\title{
Designing of Natural Scaffold Coated with Herbal Extracts for Wound Healing
}

\author{
R Sridhar Skylab* and P Pavithra \\ Department of Biomedical Engineering, Anna University, India.
}

*Corresponding author: R Sridhar Skylab, Department of Biomedical Engineering, Anna University, Sardar Patel Road, India.
Received Date: November 20, 2020

Published Date: December 22, 2020

\begin{abstract}
Skin is the most important organ of the human frame. It acts as abarrier and protection to the complete human body. When skin gets injured, the repair process entails removal of the damaged tissue and laying down of a new extracellular matrix (ECM) over which epidermal continuity can be re-established. Burns can cause many of the reasons. It can cause tremendous problems for themselves and their families. Burns are classified according to its depth of severity. Nowadays industries are moving towards the water origin collagen, particularly the fish to create a scaffold for wound treatments. Instead of deriving collagen from fish, the fish skin acts as the scaffold. Tilapia fish is rich in collagen which is used to create a scaffold to treat wounds. This is the first time that the origin the collagen is directly applied on the skin for wound healing. Now a day's modern medicines are derived from medicinal value herbs. Herbs have its own medicinal values. In this study, tridax procumbens is chosen because of its higher wound healing and reepithelialization property. The herb is extracted and blended with tilapia's skin to minimize the healing time. FTIR studies are confirmed that the components responsible for wound healing are present in both tilapia skin and extract of tridax procumbens. Structural studies are done by SEM analysis. The breaking point of that skin is found by tensile strength analysis. The biodegradation study revealed that the scaffolds mass reduced to $50 \%$ in 20 days. After 35 th day the rate of reduction in mass was very less when compared with previous days. MTT study revealed that the coated scaffold has less toxicity when compared to the coated scaffold.
\end{abstract}

Keywords: Burns; Collagen; Degradation; Scaffold; Tridax procumbens; Tilapia; Wound healing

\section{Introduction}

Many advancements are arising to treat the wounds, especially for burn wounds. Wounds that can cause psychological and economic problems in human life. However, the treatments get advanced day by day. The general classification of wounds is shown in bellow.

Now a days industries are moving towards the collagen origin, which are all cost effective to the application of wounds. Collagen type-I are responsible for fibril formation, which leads to faster healing. Wound healing contains three major phases.

\section{Phases of wound healing}

- Inflammatory (reactive)
- $\quad$ Proliferative (reparative)

- $\quad$ Maturation (remodeling) (Figure 1)

If the skin gets injured, the body responds easily. The wound healing time is depending on the vascular and cellular response of the injury. The blood vessels are constrict within a few seconds in the injured area to avoid blood loss. Platelets are accumulated in the injured area and hence clots the blood. To expose the sub endothelial collagen surface, the endothelial cells are retracted. In that surface the platelets are attached. Through the adhesive glycoproteins (fibrinogen, fibronectin, thrombospondin and von willebrand factor) the adherence occurs between exposed collagen surface and other platelets. The factors which attract other 
important cells to the injury are also released by the platelets. After that neutrophils are enter in the wound site. It used to fight against the infection, break downs the necrotic debris by attracting the macrophages and activates the fibroblast response.

The inflammatory phase remains 5 minutes to 5 days. After that it leads to the proliferation phase of the wound healing process. The primary platelet plug is formed by the aggregation of platelets. The platelets are activated by aggregation and attachment to the collagen surface. The degranulation, release of chemotactic and growth factors such as platelet-derived growth factor (PDGF), protease, and vasoactive agents are activated by enabling the platelet. In the 2 different pathways the coagulation occurs.

- Intrinsic pathway.

- $\quad$ Extrinsic pathway.
By activating the factor XII (Hageman factor) the intrinsic pathway begins. Whenever the blood is exposed to extravascular surface this factor is activated. Similarly, by activating the tissue factor the extrinsic pathway begins. Particularly the tissue factor found in the extravascular cells in the presence of factors VII and VIIa. Then both are proceeding with thrombin. The thrombin is then activated. It converts fibrinogen to fibrin. The fibrin is the important factor in the wound healing mechanism. The wound healing is impeded if the fibrin matrix is removed. As the result aggregation of platelet and coagulation cascade is clot formation. It occurs only in the site of injury.

In addition to activation of fibrin, thrombin facilitates migration of inflammatory cells to the sites of injury by increasing vascular permeability. By this mechanism, factors, and cells necessary to healing flow from the intravascular space and into the extravascular space (Figure 2).

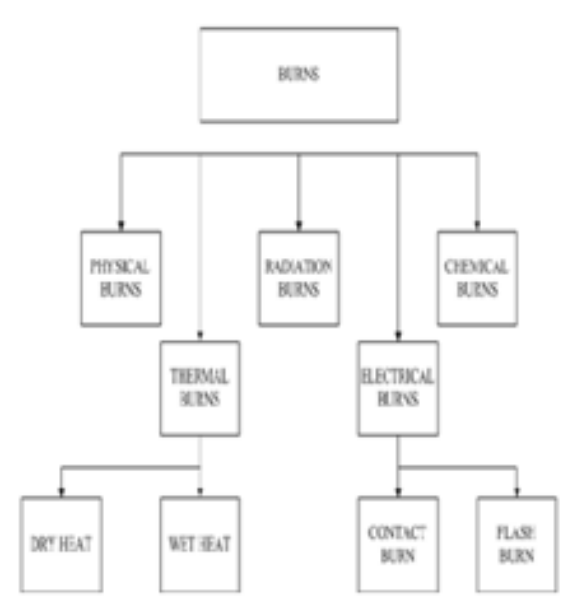

Figure1: Classification of Burn Wounds.

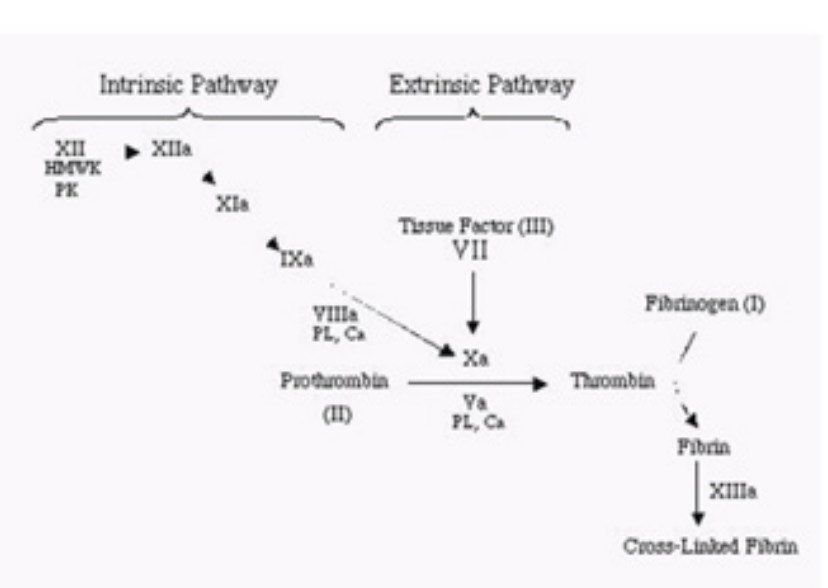

Figure2: Review of coagulation pathway.

\section{Proliferation Phase}

Proliferation phase is followed by inflammatory phase. It remains 24 to 72 hours. Mitotic activity is carried on the surface of the wound by bursting out the epidermal cells into mitotic activity. Across the surface of the wound, the cells begin to migrate. In the deeper parts of the wound the fibroblasts starts to proliferate. 
Small amounts of collagen are synthesized by these fibroblasts and it helps further fibroblast proliferation by acting it as a scaffold. Granulation tissue consists of capillary loops supported in this developing collagen matrix, also appears in the deeper of the wound. Fibroblastic phase is carried out in this phase. Depending upon the seviourity of injury, after four to five days, fibroblasts begins to produce large amounts of collagen and proteoglycans.

Collagen fibres are laid down randomly and are cross-linked into large, closely packed bundles. Proteoglycans are used to enhance the formation of collagen. In some injuries this phase remains up to few months. After 15 to 20 days of this phase it enters into maturation phase.

\section{Maturation Phase}

In this phase the collagen is remodeled into more organized manner. Tensile strength increased within one year depending on the seviourity of the wound. They may regain the original strength by 70 to $80 \%$. After this phase the scar may formed.

While treating second and third degree burn wounds, pain causes during the dressing. To avoid that single time, use of natural scaffold is used to treat it. The scaffold is made from collagen rich fish skin. Collagen type-I plays a vital role in wound healing in the remodelling phase. Collagen type-I is abundantly available naturally in animals like fish. Particularly in fish bone, skin and tendon has high collagen. And it is easily available everywhere. So the fish skin is used as a scaffold. In this work the tilapia fish is used as a scaffold material. Because it is rich in collagen type-I when compared to other fishes. It is easily available fish. Its cost is less. Because of those reasons that fish is chosen. Indian plants have many number medicinal values. Tridax procumbens is one among that. It is commonly known as Jayanti Vedain Sanskrit and in English popularly called as 'coat buttons'. The plant is native of tropical America and naturalized in Africa, Asia, Australia and India. The whole plant (weed, stem, leaf and flower) has a medicinal property. Especially have a wound healing property. It is distributed in all the areas, and available in all the seasons.

In this work the scaffold was prepared by sterilizing the tilapia's skin and it is coated/blended with the tridax procumbens extract by using the laboratory instruments.

In the previous studies the scaffold is prepared only by sterilizing it by using specially designed equipment to it [1-5]. The collagen is derived to make a scaffold from the marine origin animals like fish because of its availability and cost. The derived collagen is especially used in wound healing application [5-10]. The herbs have its own medicinal property. And it is used for various medicinal applications. The tridax procumbens has the capability to heal the wound [5].

\section{Methodology}

(Figure 3)

\section{Separation of tilapia's skin}

(Figure 4,5) Separation was done in a careful manner. Because removal of flesh after peeling is a difficult one. In the presence of flesh, the contamination may occur. The tilapia skins were peeled off which are the origin of Indian sea water. If it has any flesh it was removed by cutting. Then it was washed in the running distilled water for three to five minutes to avoid the traces of any blood or scale [10-14].

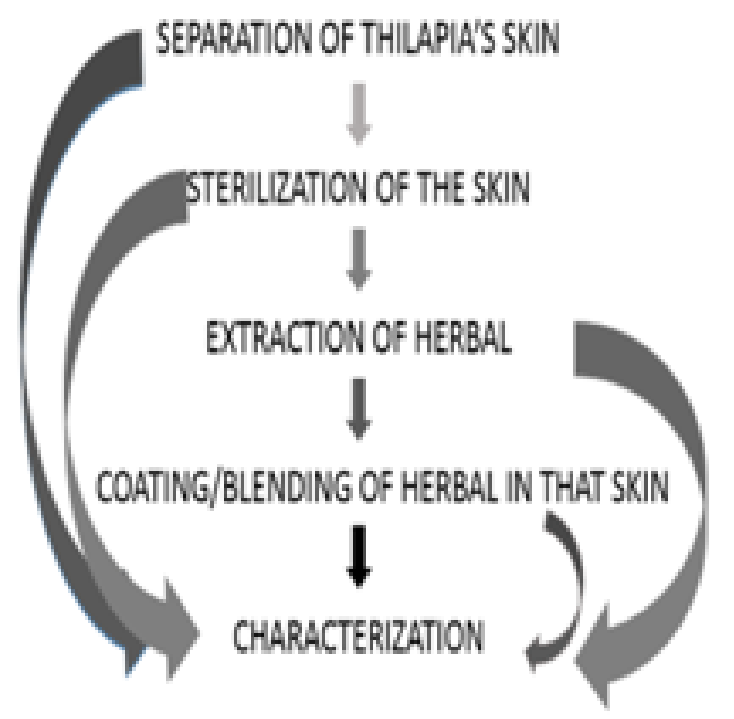

Figure 3: Methodology to develop scaffold. 


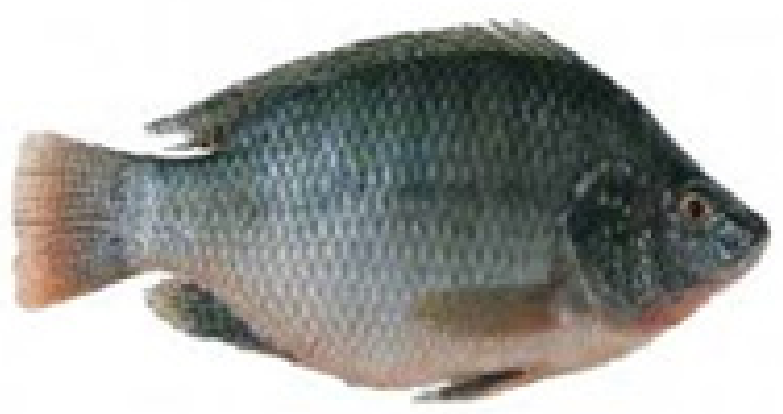

Figure 4: Tilapia Fish.

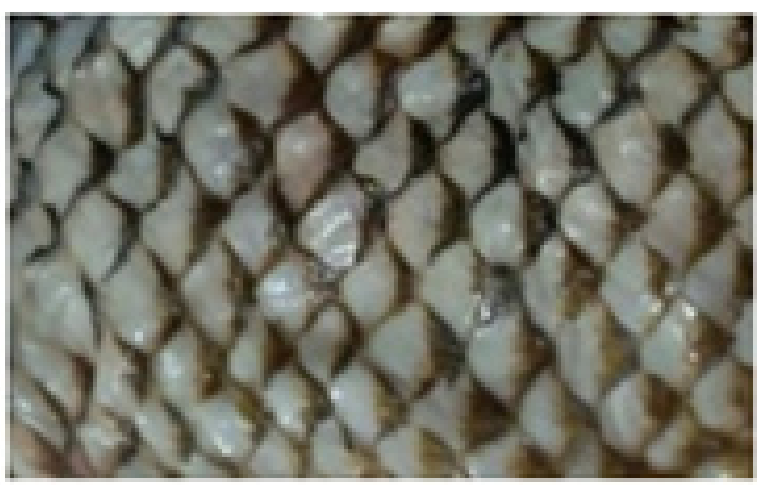

Figure 5: Separated Tilapia Fish Skin.

\section{Sterilization of the skin}

- After washing that skins were placed in the sterile container containing $2 \%$ chlorhexidine gluconate where they remain for $30 \mathrm{~min}$.

- $\quad$ After the previous operation, the skins are washed with sterile SF and removed to another container containing another $2 \%$ chlorhexidine gluconate solution, where they kept for 30 minutes.

- The skins were rinsed in sterile saline and placed in a container containing $50 \%$ glycerol solution, $49 \%$ saline solution and $1 \%$ penicillin, streptomycin and fungicide solution and kept in an ice-containing isothermal box for $3 \mathrm{hrs}$.

- The next steps are carried out in the shaker, with the horizontal flow.

- The pelts were removed from the previous solution, washed with sterile SF and placed in a sterile, sealed container containing $75 \%$ glycerol, $24 \%$ saline and $1 \%$ penicillin solution,
Streptomycin and fungicide, and held for 3 hours in a water bath, with a stirrer at a constant speed of 15 revolutions per minute and a temperature of $37^{\circ} \mathrm{C}$.

- $\quad$ The pelts are removed, washed with sterile SF again and placed in another sterile and airtight container. Containing 99\% glycerol, $1 \%$ penicillin, streptomycin and fungicide solution, and kept in a water bath at $37^{\circ} \mathrm{C}$ and 15 revolutions per minute for a further 3 hours

- $\quad$ At the end of the last stage, the pellets will be packaged in double plastic envelopes and stored at $4^{\circ} \mathrm{C}$ for later use, with a shelf life of up to two years (Figure 6).

\section{Materials Used}

- $\quad$ Chlorhexidine gluconate-ICPA HEALTH PRODUCTS LTD

- $\quad$ Sterile SF-Terbutaline Sulphate

- $\quad$ Glycerol-Sipali (I) Pharma

- $\quad$ Streptomycin-Nitin Life science LTD. 


\section{Extraction of herbal}

(Figure 7) The whole plant (stem, leaf, flower, and weed) has a wound healing property. So, the whole plant was washed in the running distilled water. After washing it is grinded to get the extract.
Then it is filtered using Whatman number 2 filter paper to remove granules. After filtering it is centrifuged for 15 minutes at $30 \mathrm{rpm}$ to get a proper extract mixture. Then it is stored in the centrifuge tube at $4^{\circ} \mathrm{C}$ for future use (Figure 8).

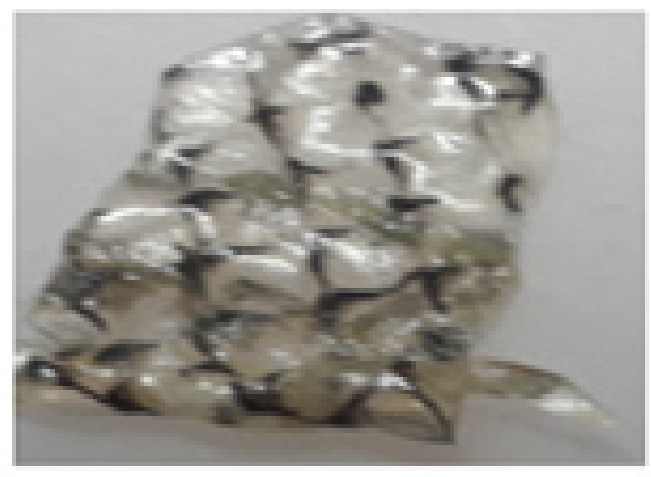

Figure 6: Sterilized Tilapia Fish Skin.

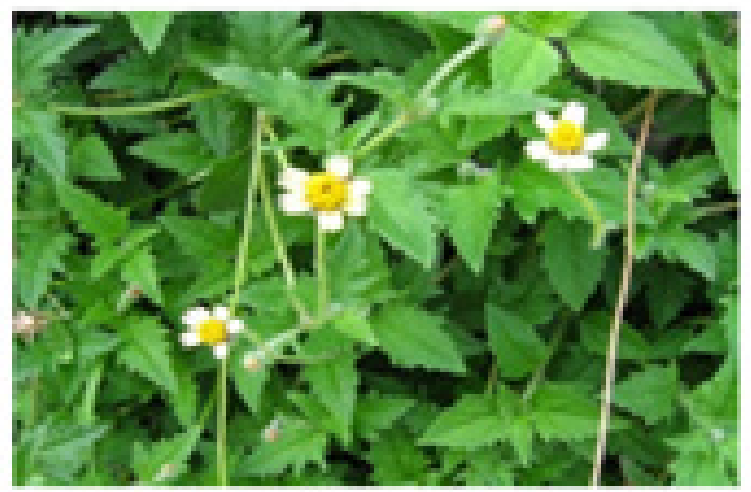

Figure 7: Tridax Procumbens Plant.

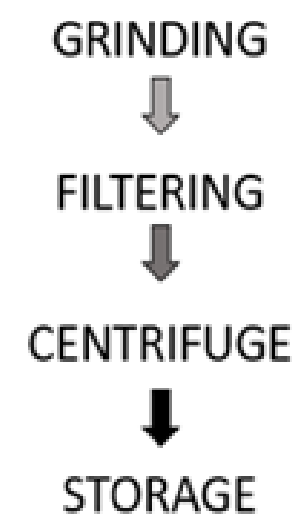

Figure 8: Extraction of herbal. 


\section{Blending of herbal in skin}

The extracted herbal was taken in the beaker along with the glycerol in the ratio of 1:1. Then the sterile tilapia skin is soaked in that mixture and it is stirred for 15 minutes in the magnetic stirrer to get the end product (Figure 9).

\section{Characterization}

- $\quad$ FTIR

- Mechanical strength

- $\quad$ SEM analysis

- $\quad$ Cell viability

- Biodegradability

\section{FTIR}

FTIR (Fourier Transform Infra-Red) is the preferred method of infrared spectroscopy. When IR radiation is passed through the sample some radiation is absorbed by the sample and some of them passed through (is transmitted). The resultant gives the fingerprint of the sample. The uses of FTIR is, converts the detector output into an interpretable spectrum. Generates the spectra with patterns that provide structural insights. The interpretation table helps to analyses the spectrum (Table 1).

\section{Mechanical strength}

In this work, the tensile strength was found in the mechanical strength. Tensile strength is the stress at which a force applied causes the material to lengthen then break. For an axial load material, the breaking strength in tension is $\mathrm{s}=\mathrm{P} / \mathrm{a}$ where $\mathrm{s}$ is the breaking strength, $\mathrm{P}$ is the force that can cause it to break and a is the cross- sectional area. The tensile strength is dependent on the type of material and the cross- sectional area.

\section{SEM analysis}

SEM (Scanning Electron Microscope) scans a focused electron beam over a surface to create an image. The electrons in the beam interact with the sample, producing various signals that can be used to obtain information about the surface topography and quantitative composition. It gives the high-resolution images and precisely measures the small feature of the sample.

Cell viability is a viability assay is an assay to determine the ability of organ, cells or tissues to recover its viability. It is used to measuring the results of cell proliferation, cell death and cytotoxicity of the sample. In this proposed system MTT assay method is used. It is the high accuracy as well as less hazardous.

\section{Biodegradability test}

The biode gradation method is usually to predict the degradation of a particular sample in the environment. Many factors that may affect the degradation. The aim is to apply the end product in the human skin. So the biodegradability is done in the three different fluids (tap water, distilled water, and saline). Because our body contains higher fluids. Finally, the results were compared with these fluids.

\section{Result and Discussion}

\section{F FTIR}

In each of the steps to observe the changes of components present in the samples were observed by FTIR analysis. Collagen is the major component in the wound healing which is present in the tilapia skin. It was discussed in this section detailed.

\section{Herbal Extract}

The herb here used was tridax procumbence. It has lysine, glycine, proline (has N-H stretch) and alkaloids, carotenoids and flavonoids (has $\mathrm{C}=0$ stretch) components which have the wound healing property and also the reepithelization property. The FTIR shows that the stretches present in the ranges of 3500-3000 and 1700-1600. It means that components are present in that herb. Due to the presence of water in that herb, the stretch is in 3311.06. The transmittance and absorbance of the fresh herbal extract are shown below (Figure 10,11).

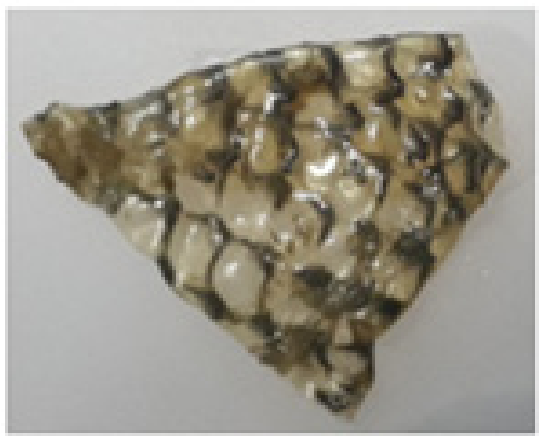

Figure 9: Coated Herbal Extract in Sterilized Tilapia Skin. 


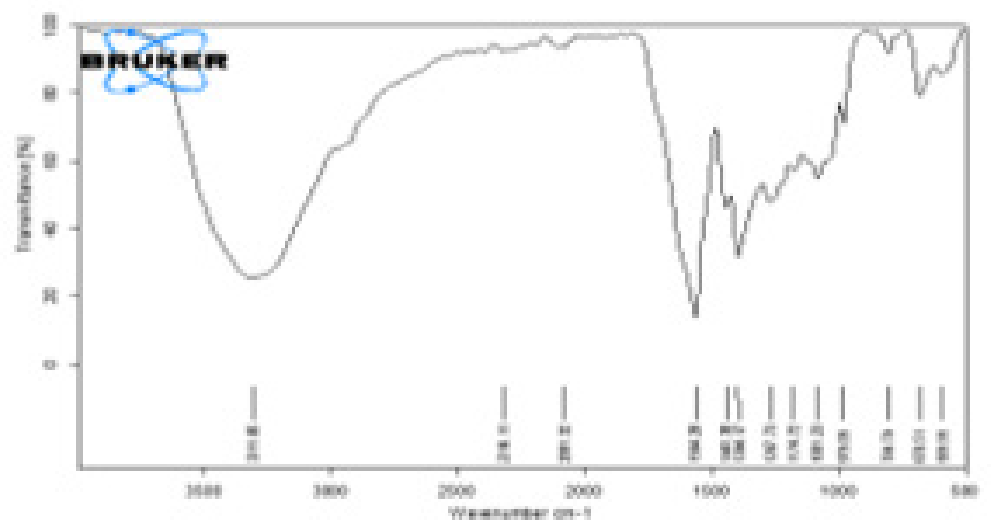

Figure 10: Transmittance of fresh herbal extract

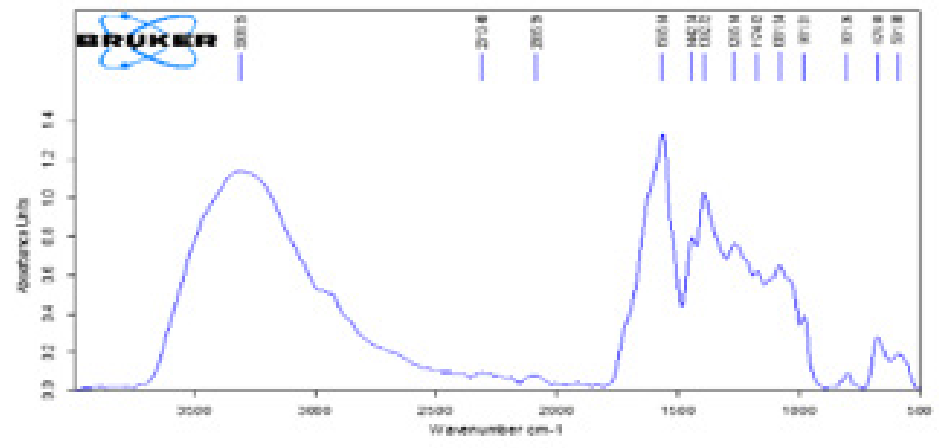

Figure 11: Absorbance of Fresh Herbal Extract

\section{Stored Herbal Extract}

The extracted herbal was stored for one week. Then it was analyzed to ratify that the components present in that herb do not vanish. The stretches which are present in the fresh herbal extract are present in the stored herbal extract. It shows that the even though the herb was stored there is no change of components in that herb. The transmittance and absorbance of the stored herbal extract shown below (Figure 12,13).

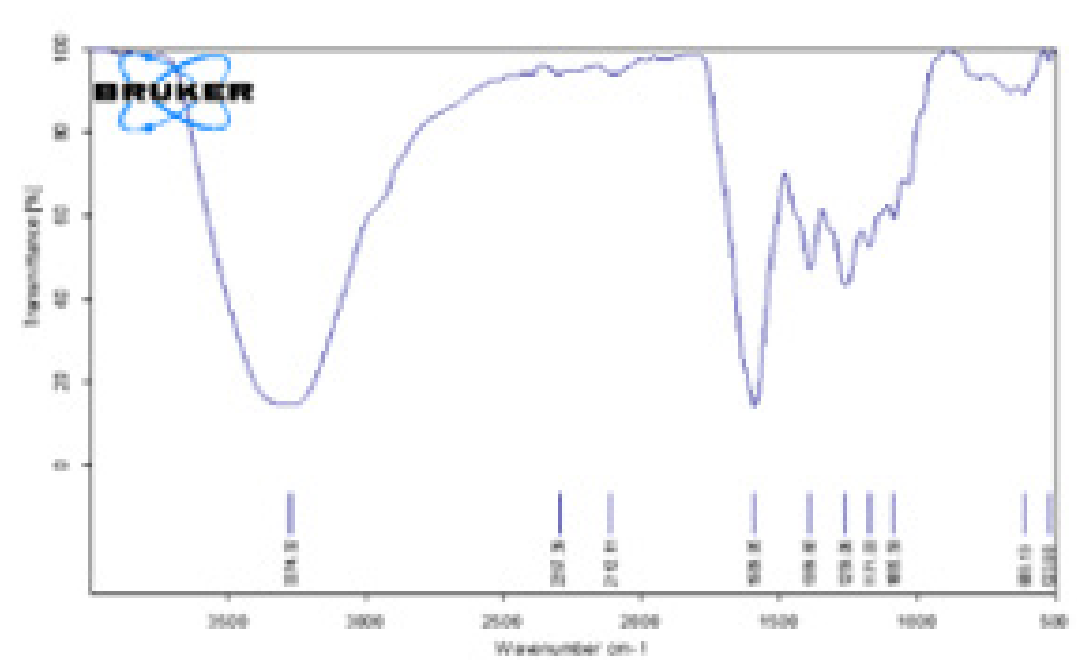

Figure 12: Transmittance of Stored Herbal Extract. 


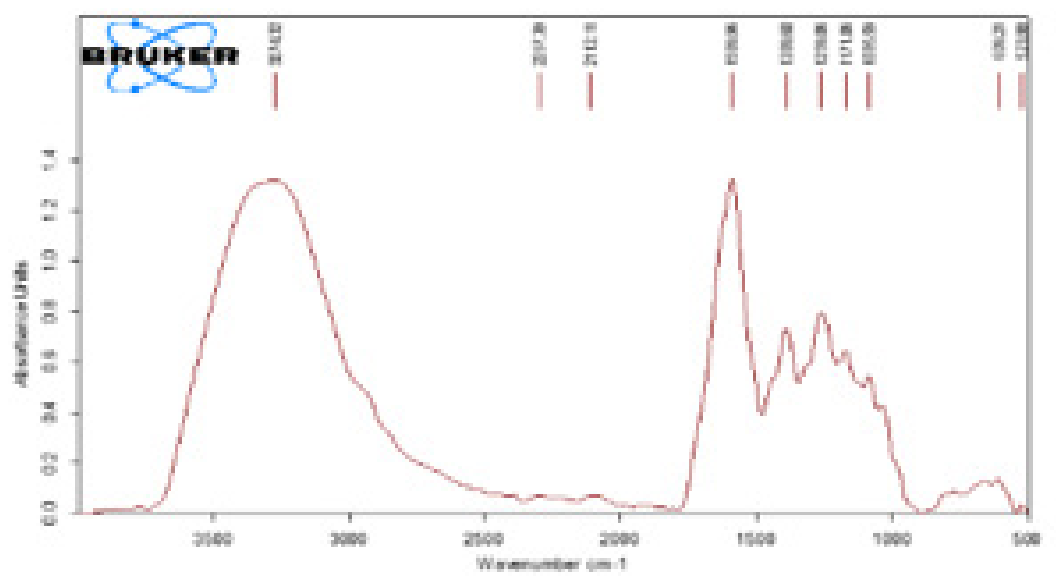

Figure 13: Absorbance of Stored Herbal Extract.

\section{Comparison of Stored and Fresh Herbal Extract}

It is proved that the two resultants are same. The brown color represents the FTIR result of transmittance of fresh herbal extract and the red color shows the transmittance of stored herbal extract. The comparison of transmittance of fresh herbal extract and stored herbal extract is shown below (Figure 14).

\section{Raw Tilapia Skin}

The reason to choose the tilapia skin was rich in collagen content. After peeling the tilapia skin it was analysed using FTIR to prove the presence of collagen type-I. Transmittance and absorbance of raw tilapia skin shown in the below figure. A stretch is present in 3284.29, it shows that the presence of N-H stretch. Means that the collagen type-I has this stretch. The broadness refers to the quantity of that component. Another stretch is in 1638.12, it shows the presence of ketones group. Glycine is one of the components in collagen, which has $\mathrm{C}=0$ stretch (Figure 15,16 ).

\section{Sterilized Tilapia}

After sterilizing the tilapia skin the FTIR was taken. The stretches are all same as the result of raw tilapia skin. It implies that the components present in the raw tilapia are not diminished even after sterilizing. The band is broader when compared to raw tilapia skin. It is due to the use of glycerol in the sterling process. The transmittance and absorbance of the sterilized tilapia shown below (Figure 17,18).

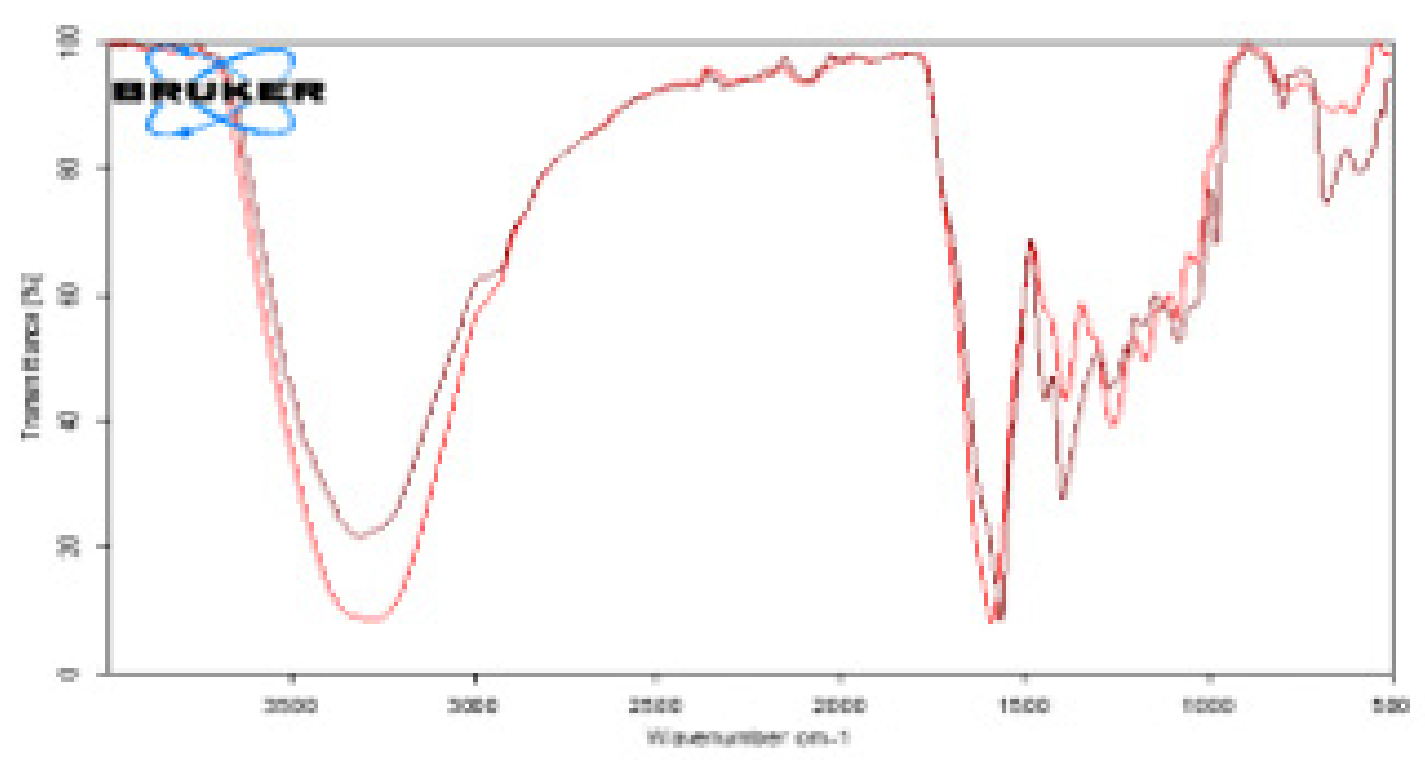

Figure 14: Comparison of Transmittance of Fresh and Stored Herbal Extract. 


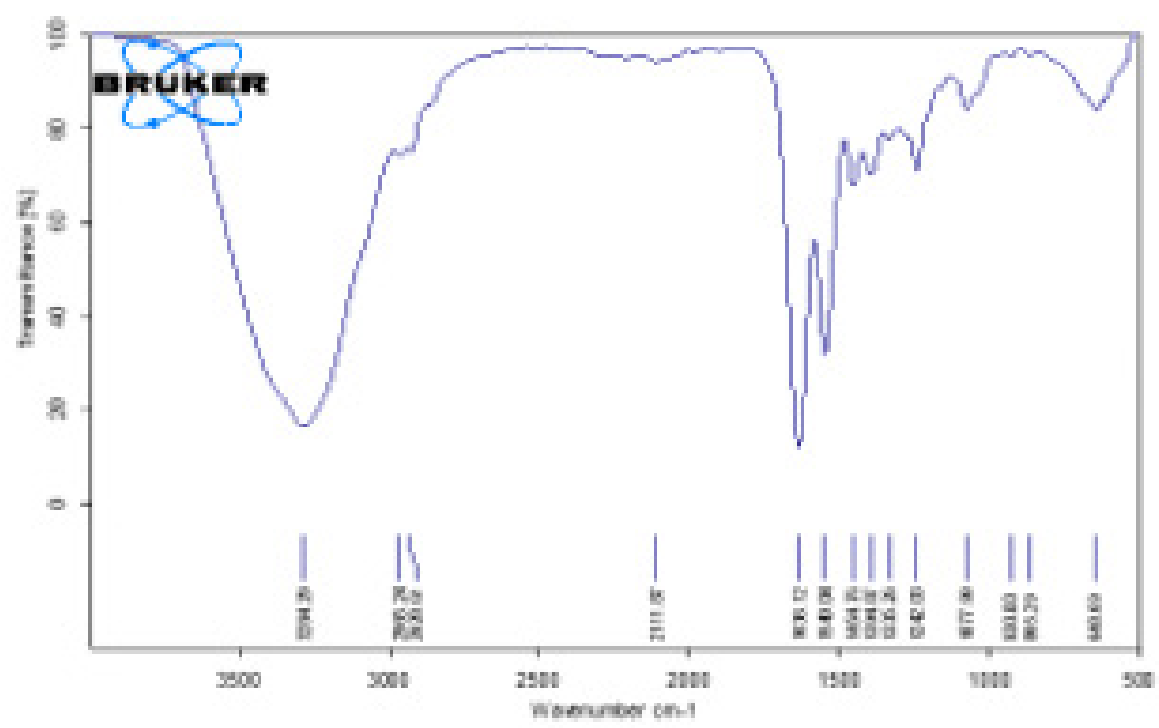

Figure 15: Transmittance of Raw Tilapia Skin.

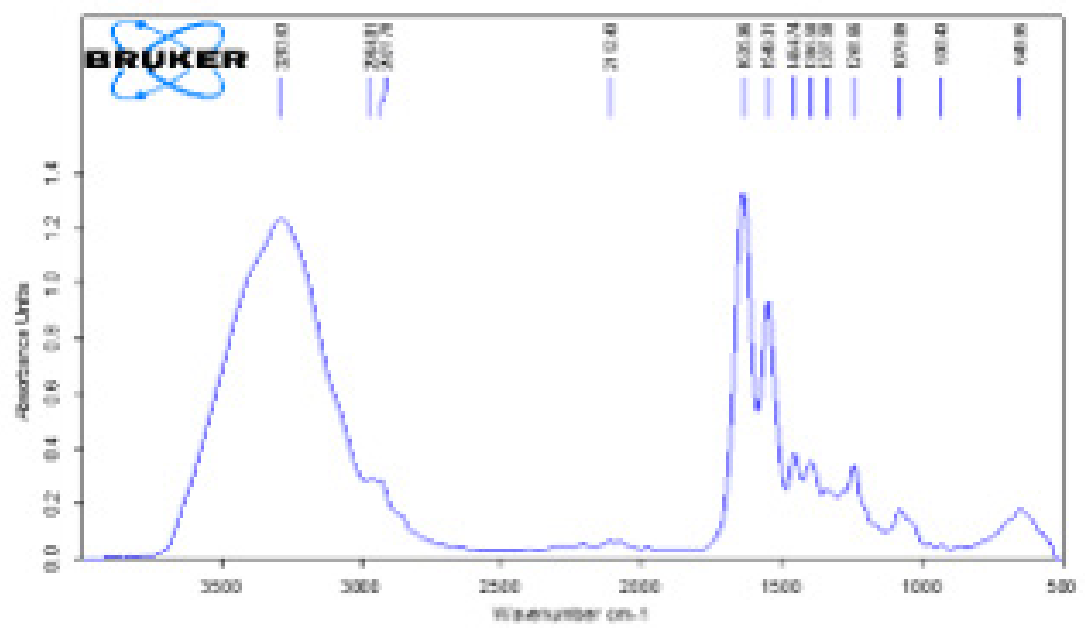

Figure 16: Absorbance of Raw Tilapia Skin.

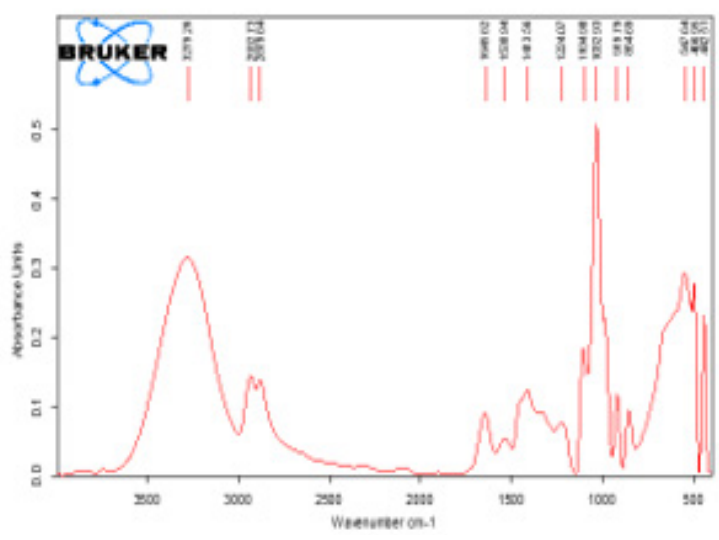

Figure 17: Transmittance of Sterilized Tilapia. 


\section{Stored Tilapia Skin}

The sterilized tilapia skins were stored at $4^{\circ} \mathrm{C}$ for a week. After it was analyzed with FTIR, to check the presence of components in that. Even though it is stored the components present in that are same as the sterilized tilapia skin. It is ratified by the FTIR results. The transmittance and absorbance of stored tilapia skin are shown below (Figure 19,20).

\section{Sterile Tilapia Blended with Herbal Extract}

The sterile tilapia skins were blended with the herbal extract in the beaker with glycerol in the ratio of $1: 1$ by the use of magnetic stirrer for 15 minutes. After that, it was analyzed by FTIR. The transmittance and absorbance of the sterile tilapia blended with herbal extract are shown in below (Figure 21,22).

\section{Comparison of Sterilized Tilapia Skin with Blended Tilapia} Skin

The herbal coated tilapia skin has higher and more component when compared to sterilised tilapia skin. The rose color line represents the transmittance of sterilized tilapia skin while blue colored spectra represent the herbal coated sterilized tilapia. Note that in that spectra no stretches are missed when compared with the previous one. The transmittance and absorbance comparison of sterilized tilapia skin with herbal coated tilapia skin is shown in below (Figure 23,24).

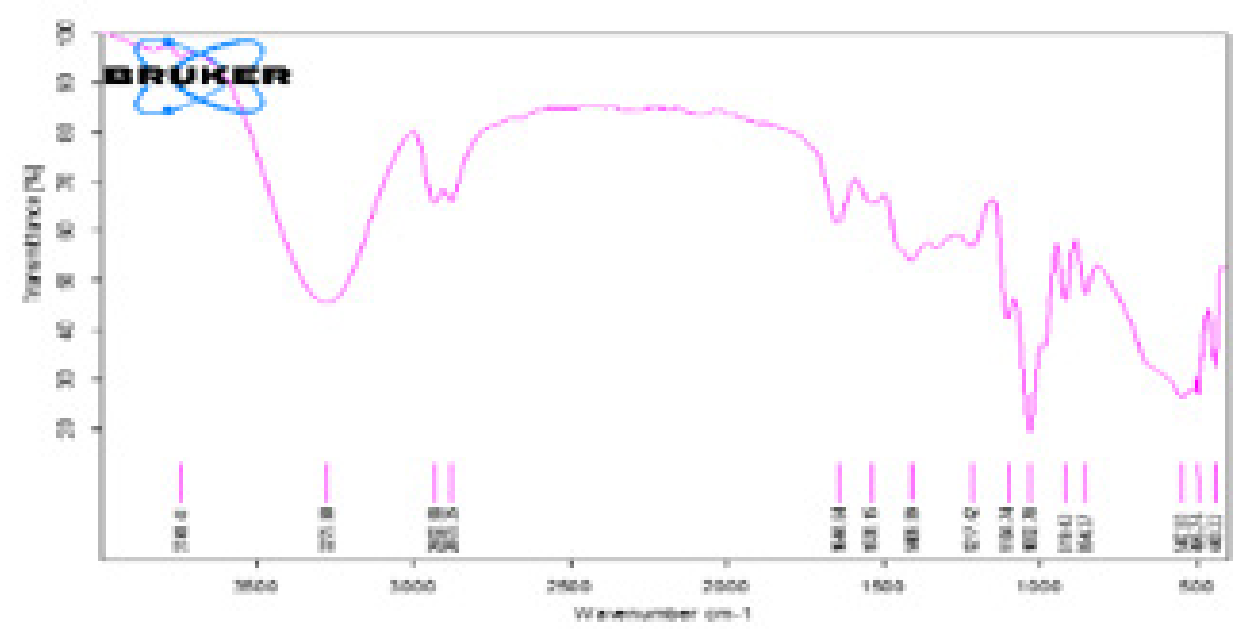

Figure 18: Absorbance of Sterilized Tilapia.

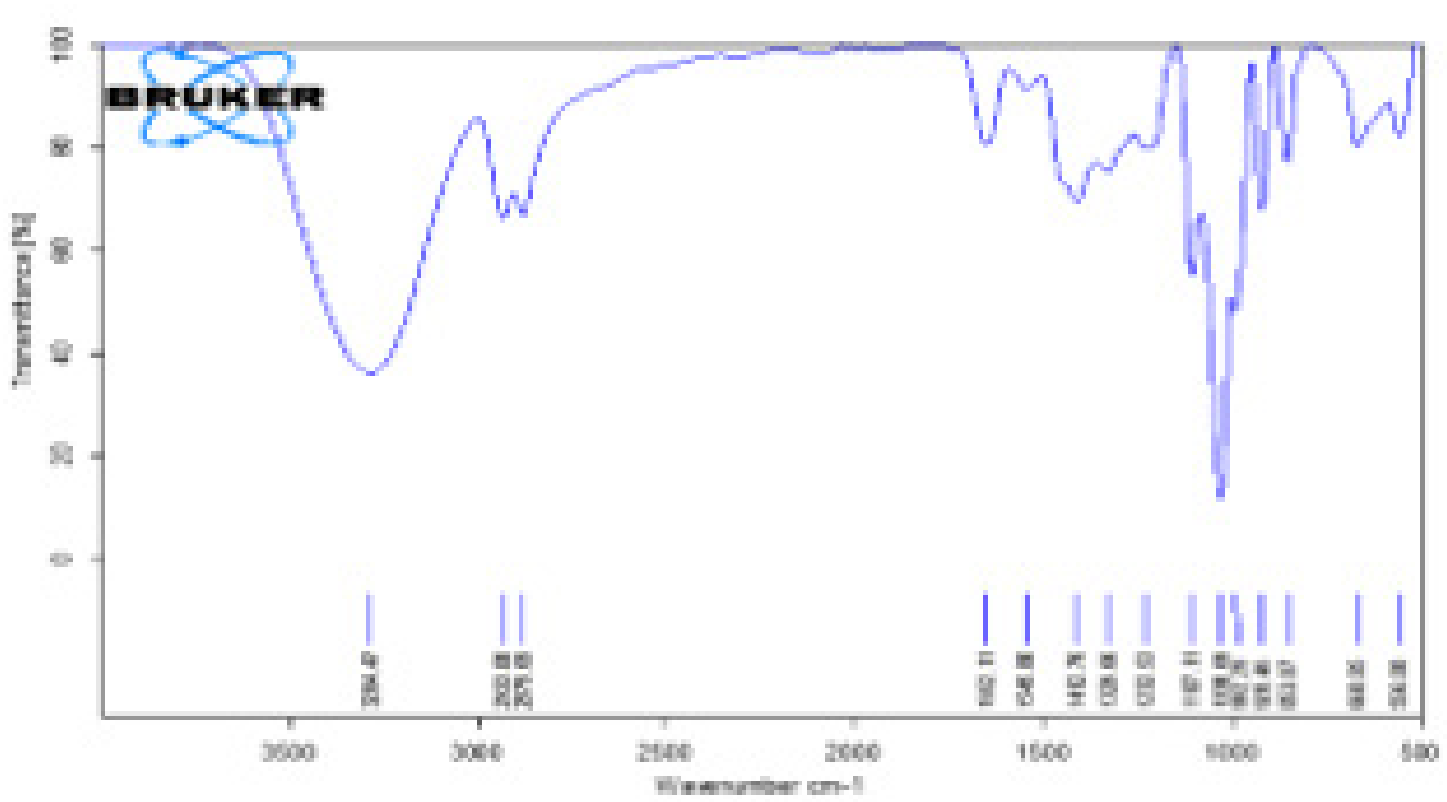

Figure 19: Transmittance of Stored Tilapia Skin. 


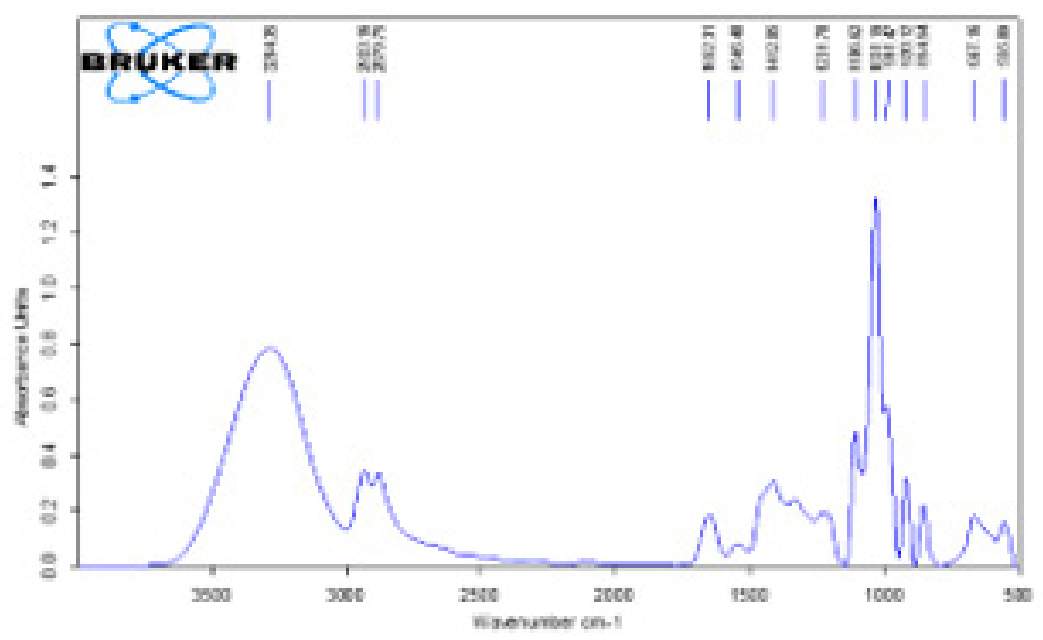

Figure 20: Absorbance of Stored Tilapia Skin.

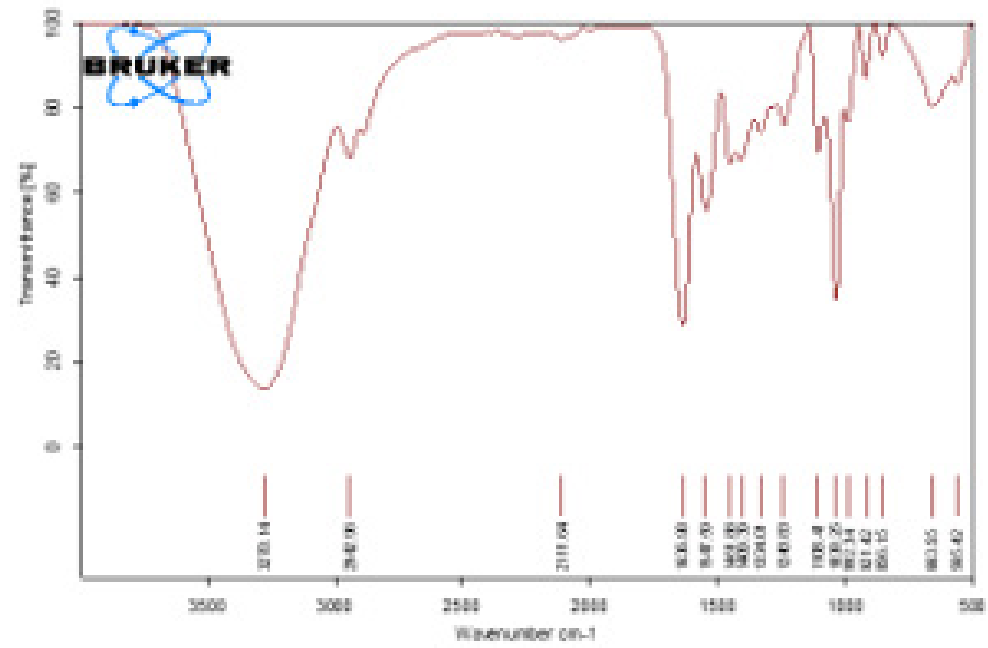

Figure 21: Transmittance of Sterilized Tilapia Skin Blended with Herbal Extract.

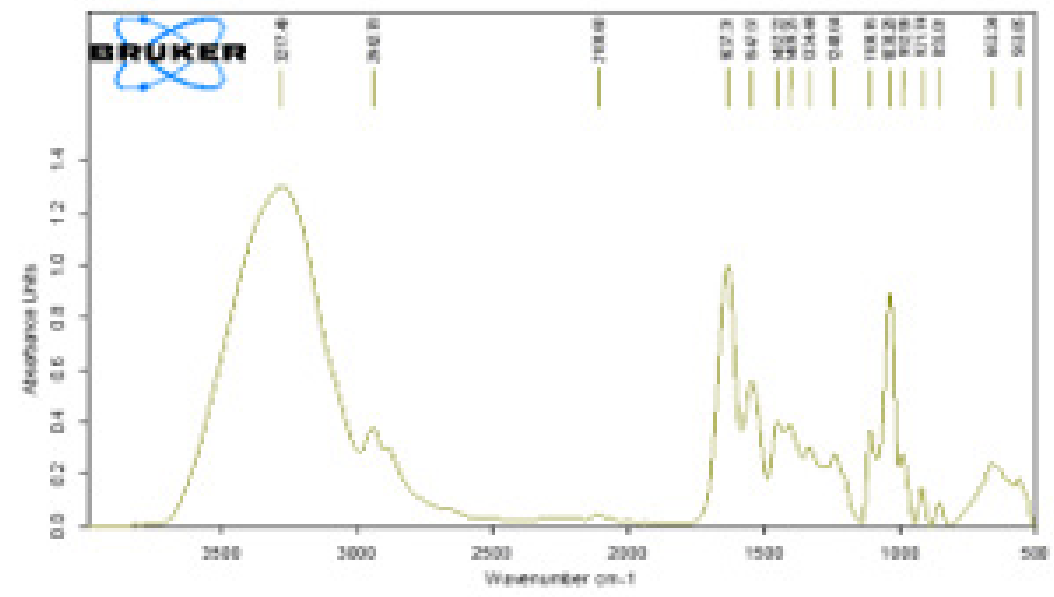

Figure 22: Absorbance of Sterilized Tilapia Skin Blended with Herbal Extract. 


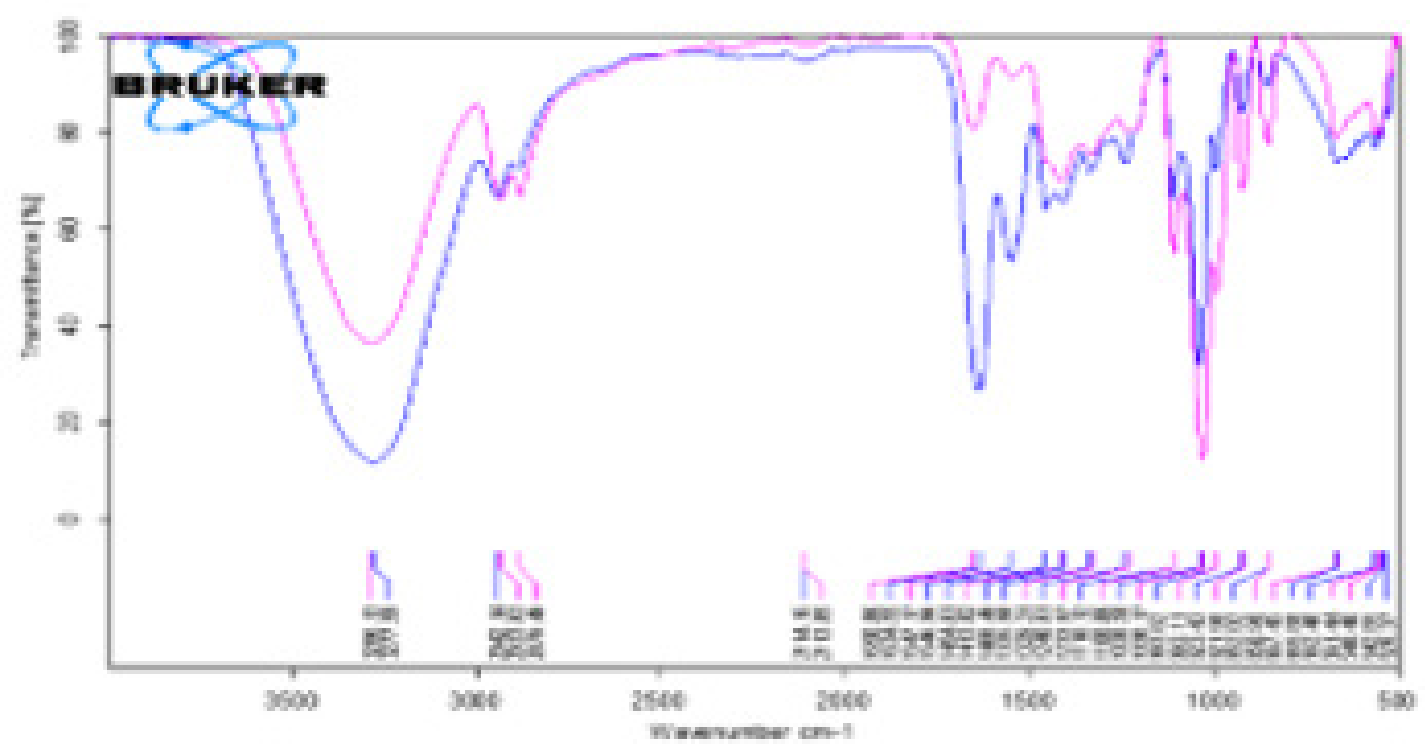

Figure 23: Comparison of Sterilized Tilapia Skin with Herbal Coated Tilapia Skin.

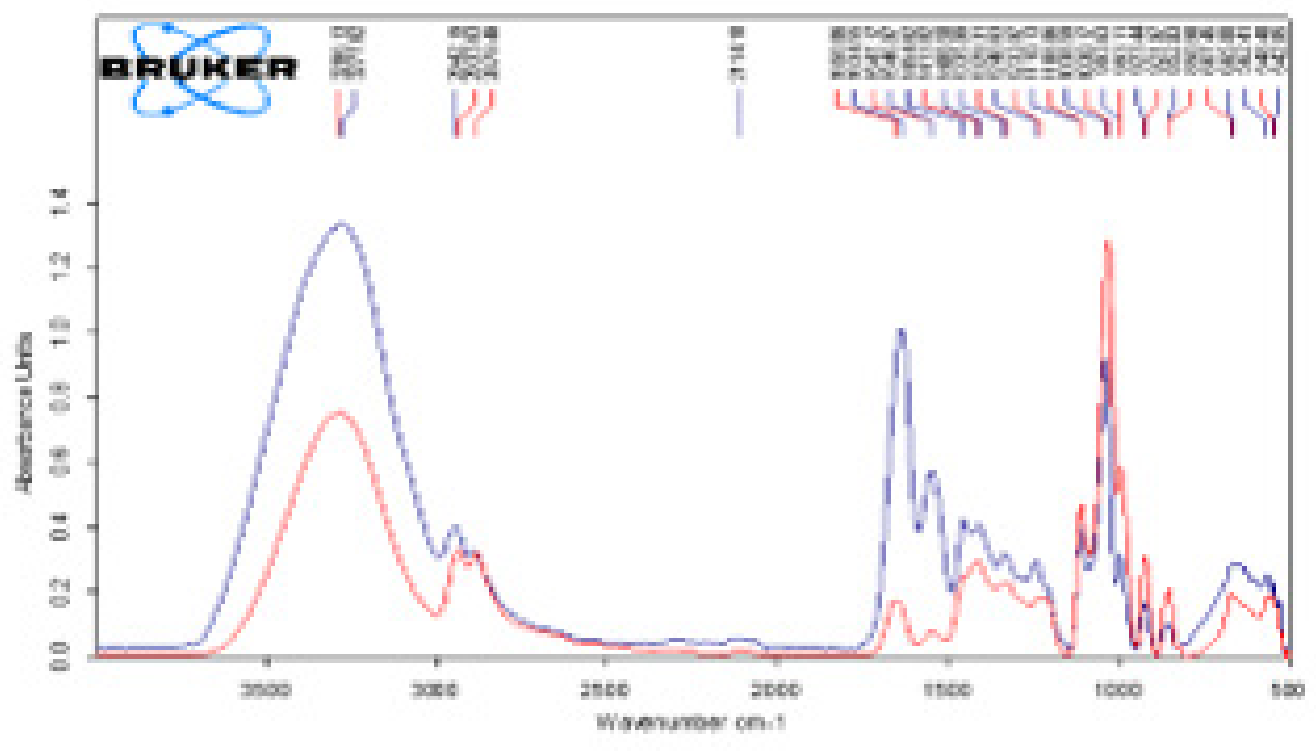

Figure 24: Absorbance of Comparison of Sterilized Tilapia Skin with Blended Tilapia Skin.

\section{Transmittance of Stored Tilapia's Skin}

The prepared scaffold was stored at $4^{\circ} \mathrm{C}$ for 6 months. Its transmittance was observed by FTIR, and it is compared with figure 18. Even though the scaffold was stored the compounds present in that scaffold are not changed (Figure 25,26).

\section{Tensile Strength}

In this work tensile strength is measured to know the breaking point of the sterilized tilapia skin coated with herbal extract. The tensile strength of the sample is calculated by using the formula

$\mathrm{S}=\mathrm{F} / \mathrm{a}$
Where, $\mathrm{S}=$ tensile strength of the sample.

$$
\begin{aligned}
& \mathrm{F}=\text { applied force to the sample. } \\
& \mathrm{a}=\text { area of the sample. }
\end{aligned}
$$

The maximum tensile strength/tensile strength of the material is

$$
\begin{aligned}
& S=0.717 / 1 \mathrm{~cm} \\
& =0.717 \mathrm{~N} / \mathrm{m}^{\wedge} 2
\end{aligned}
$$

Which is higher than the medical tape's tensile strength (Figure 27). 


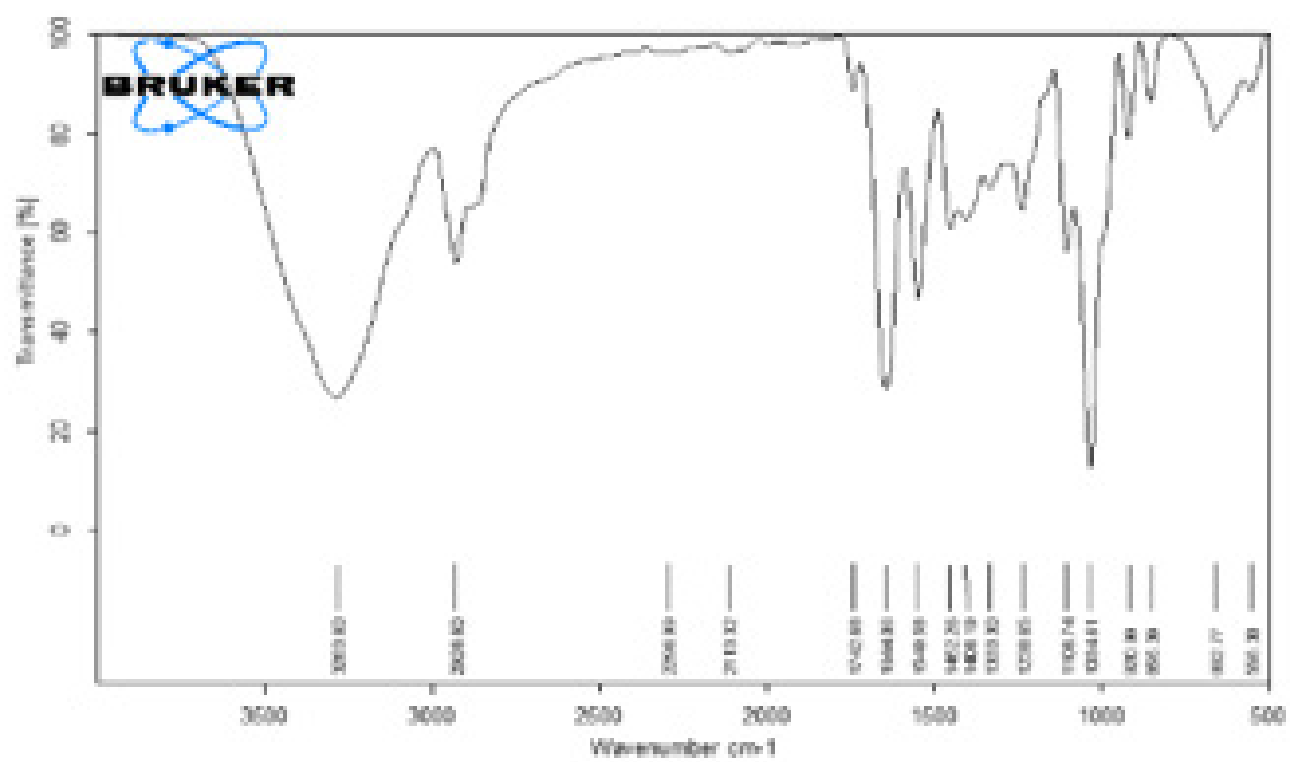

Figure 25: Transmittance of Stored Uncoated Tilapia Skin.

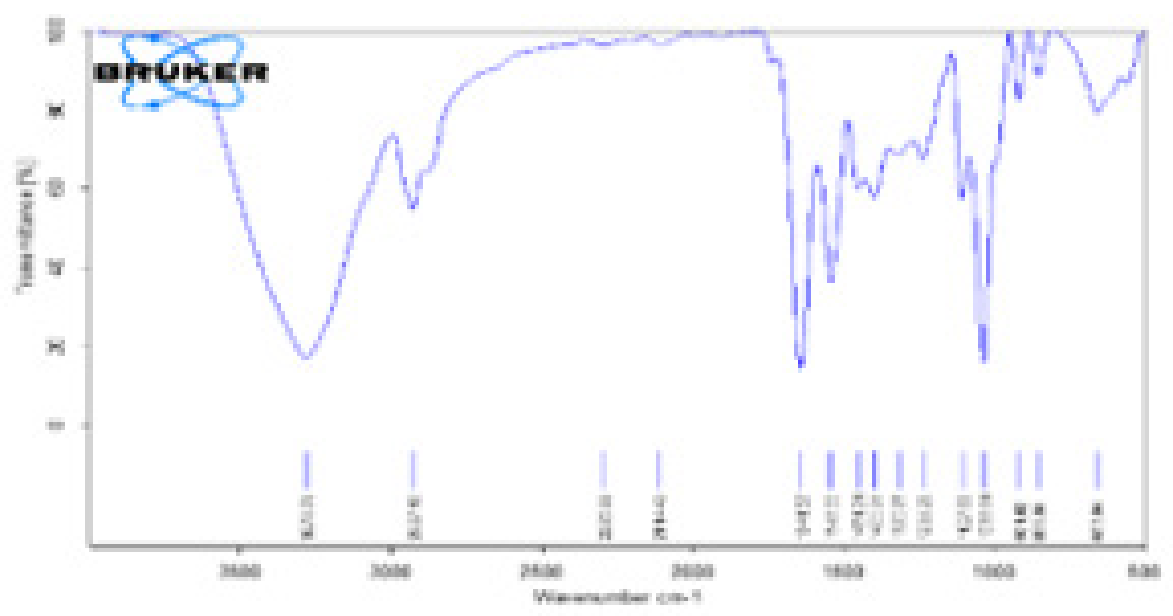

Figure 26: Transmittance of Stored Coated Tilapia Skin.

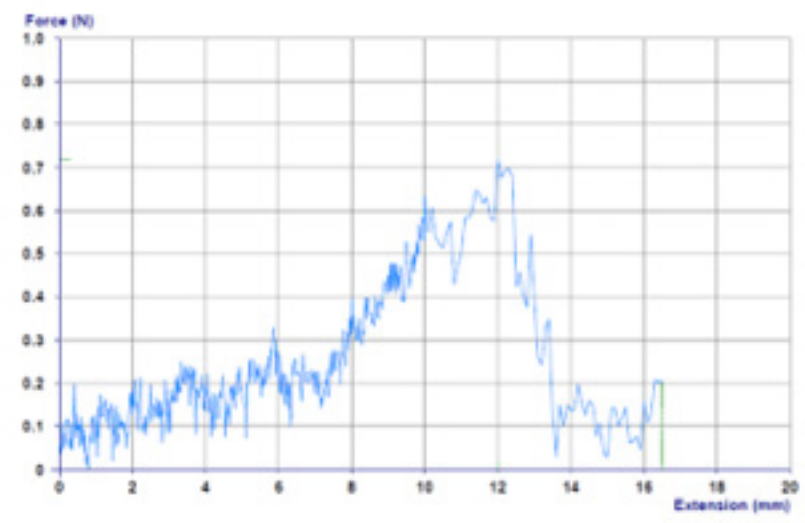

Figure 27: Tensile Strength. 


\section{Sem Analysis}

SEM (Scanning Electron Microscope) analysis is used to study the surface information of the particular sample. It gives the resolution of micrometers level. Myofilaments jointly give the fibrils; fibrils joint together give the fiber and fibre jointly gives the fiber bundles. Those fiber bundles are shown in the first image. And a small dot denotes the sponges. The various magnification level of microscopic images is shown in below (Figure 8(a-f)).

In figure $21(\mathrm{c})$ the fiber bundles are shown clearly.

\section{Cell Viability}

The viability of the cells is tested using this MTT assay method. To prepare the Extract, Sterile fish-skin samples were weighed and cut into small pieces, aseptically. These pieces were then homogenized in DMEM to get the extract of the desired concentration $(500 \mathrm{mg} / \mathrm{ml})$.

\section{MTT Assay}

EA. hy926 cells were grown to about $70 \%$ confluence in 24 well plates (Majumder et al 2008). The cells were then subjected to extracts $(200,100 \& 10 \mathrm{mg} / \mathrm{ml})$ obtained from the coated and uncoated skin samples. After treatment, the cells were washed twice with 1x PBS before being incubated with $0.5 \mu \mathrm{g} / \mathrm{ml}$ of MTT (3-(4, 5-dimethylthaizole-2-yl)-2, 5-diphenyl tetrazolium bromide) for $4 \mathrm{hrs}$. The purple- colored product formed was then dissolved with DMSO and the optical density was measured at $575 \mathrm{~nm}$ using Varian Cary 4000 UV-Vis spectrophotometer. The result of MTT assay is shown in below graph (Figure 29).

The pictorial representation of the above graph is shown in below

\section{Control}

(Figure 30, 31)

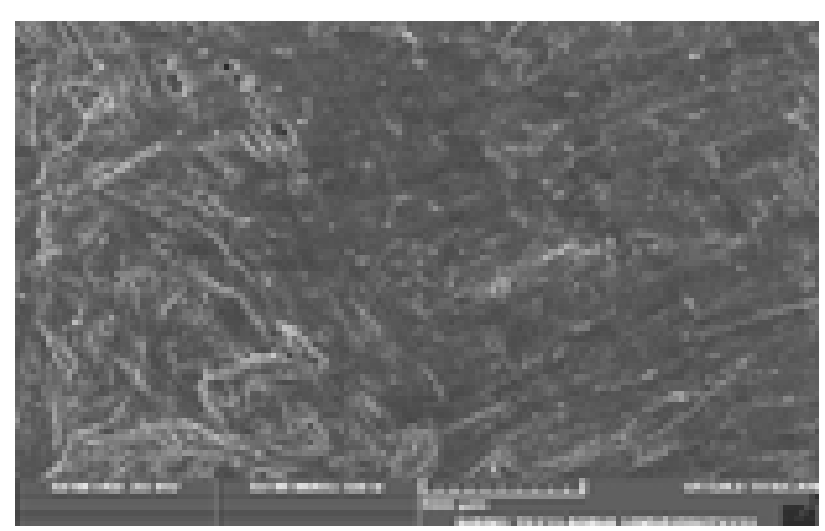

Figure 28: SEM images in various magnification levels.

Figure 28: (a)In 50x magnification.

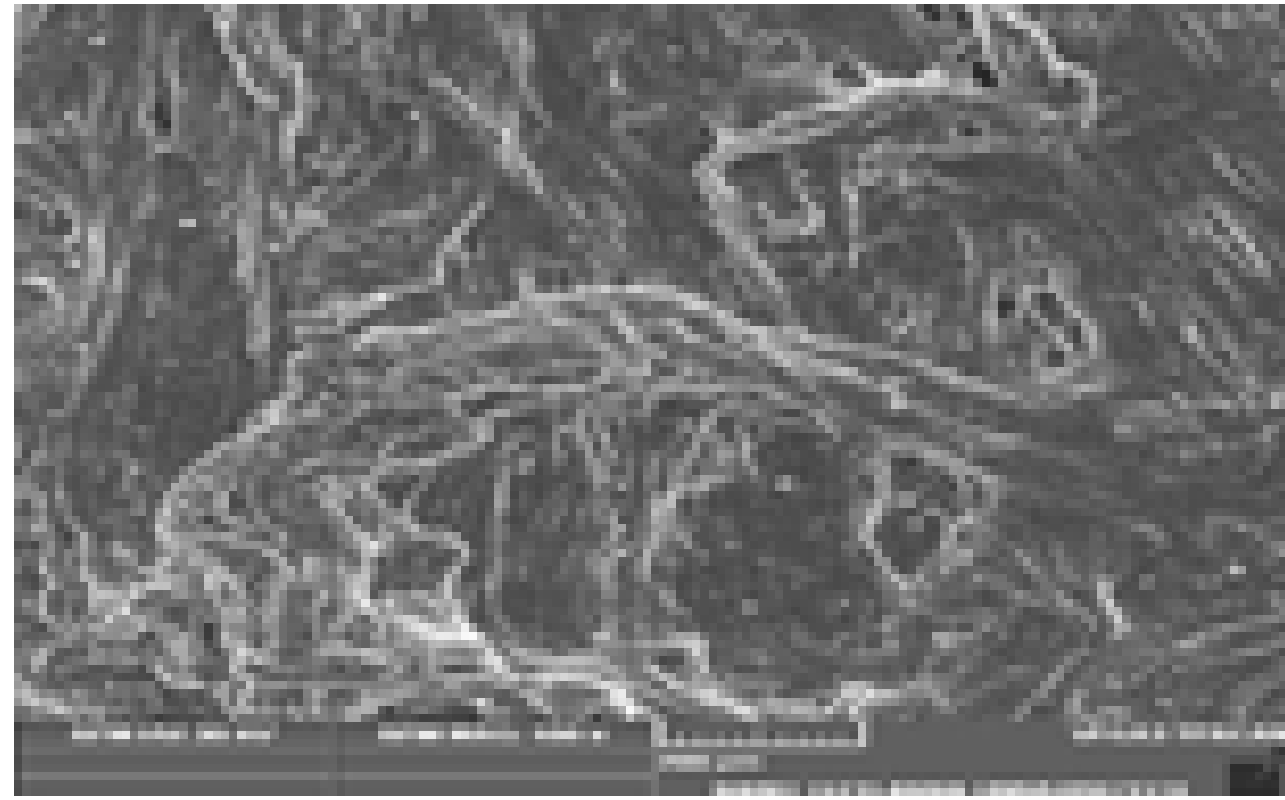

Figure 28: (b) In 100x magnification. 


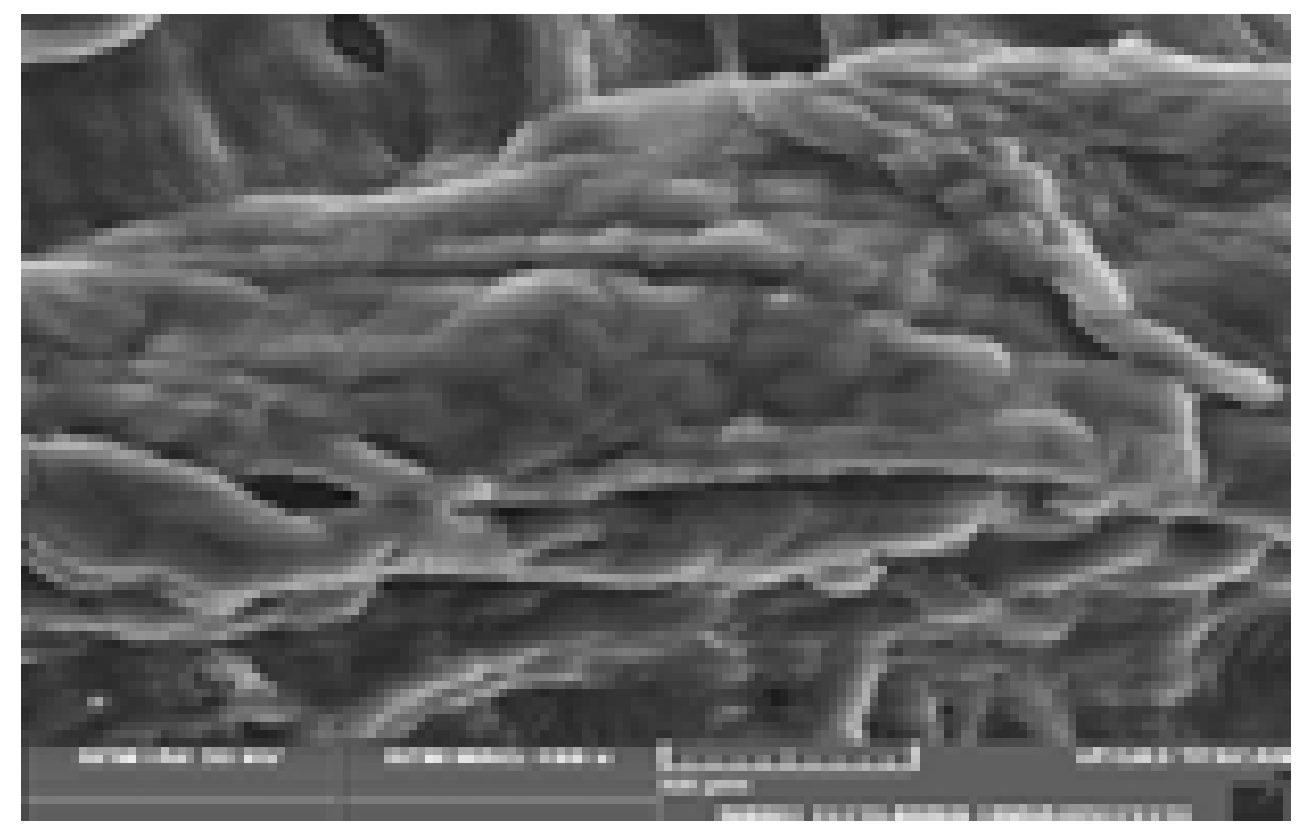

Figure 28: (c) In 499x magnification.

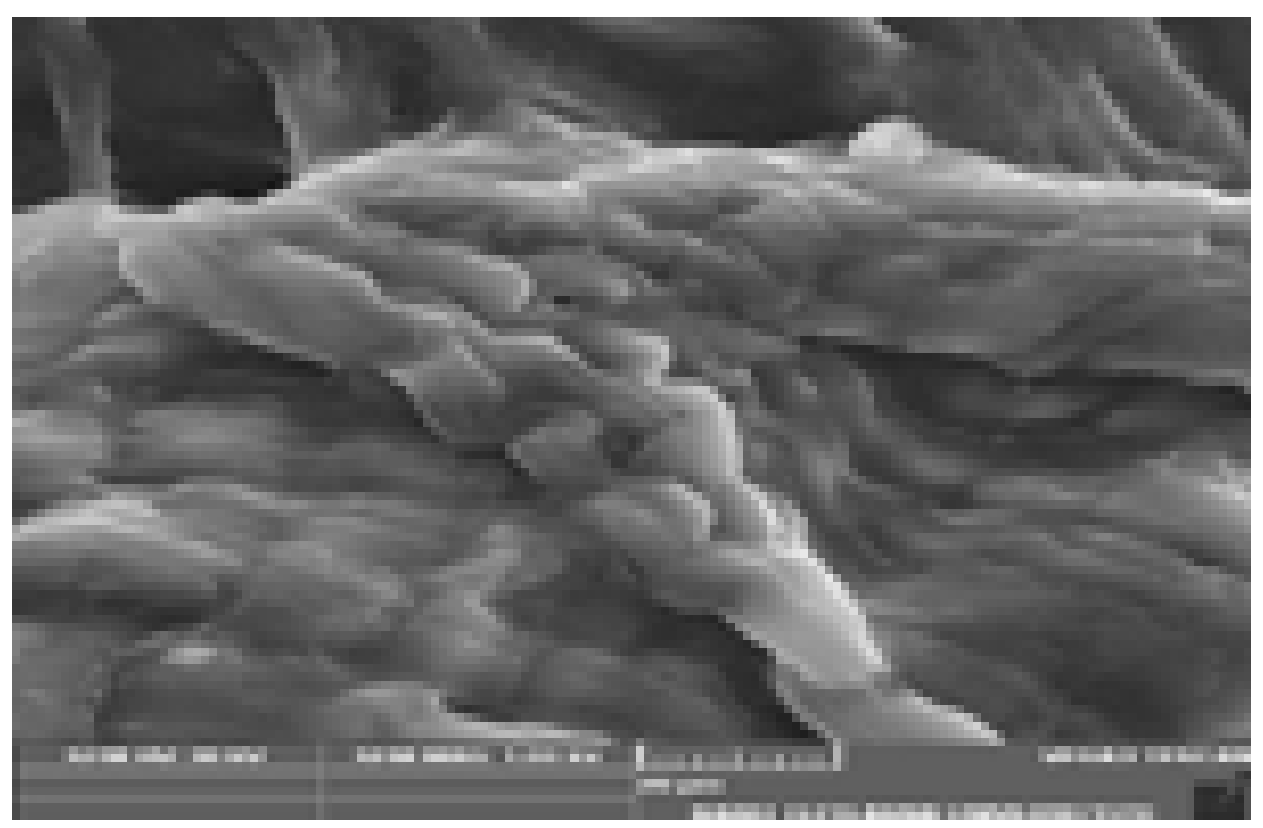

Figure 28: (d) In 1Kx magnification

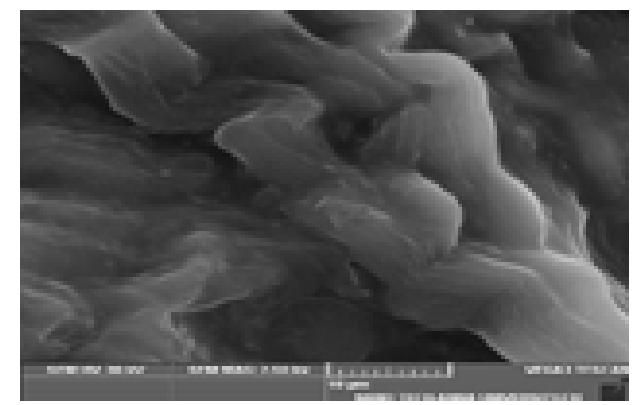

Figure 28: (e) In 2.5Kx magnification. 


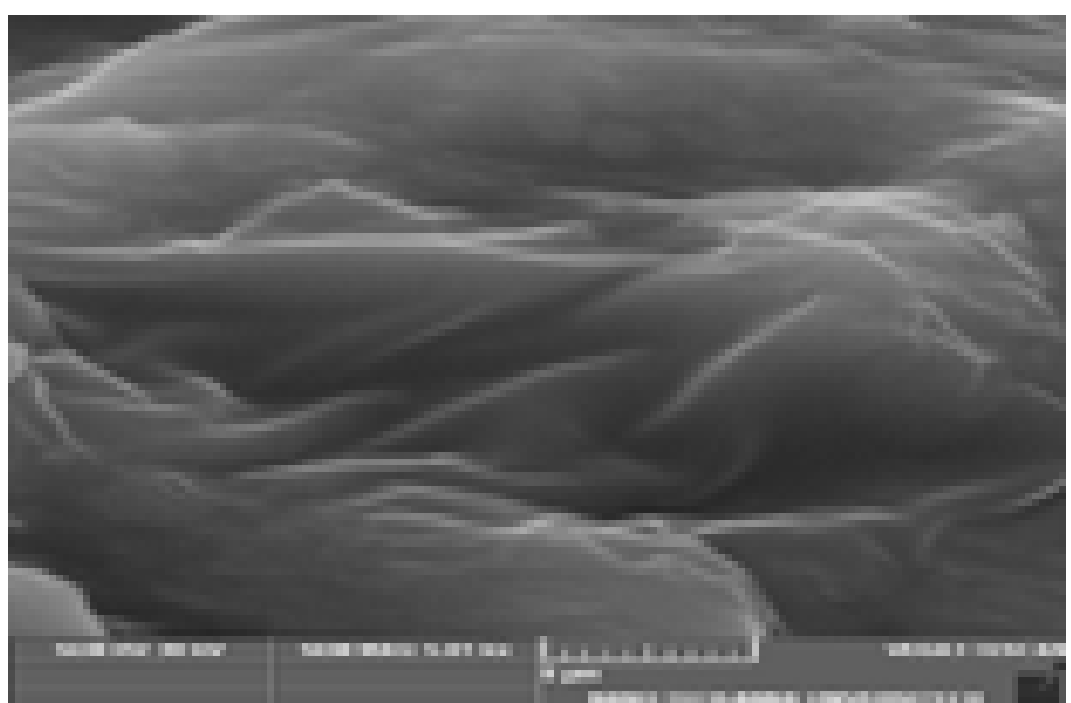

Figure 28: (f) In 5.01Kx magnification.

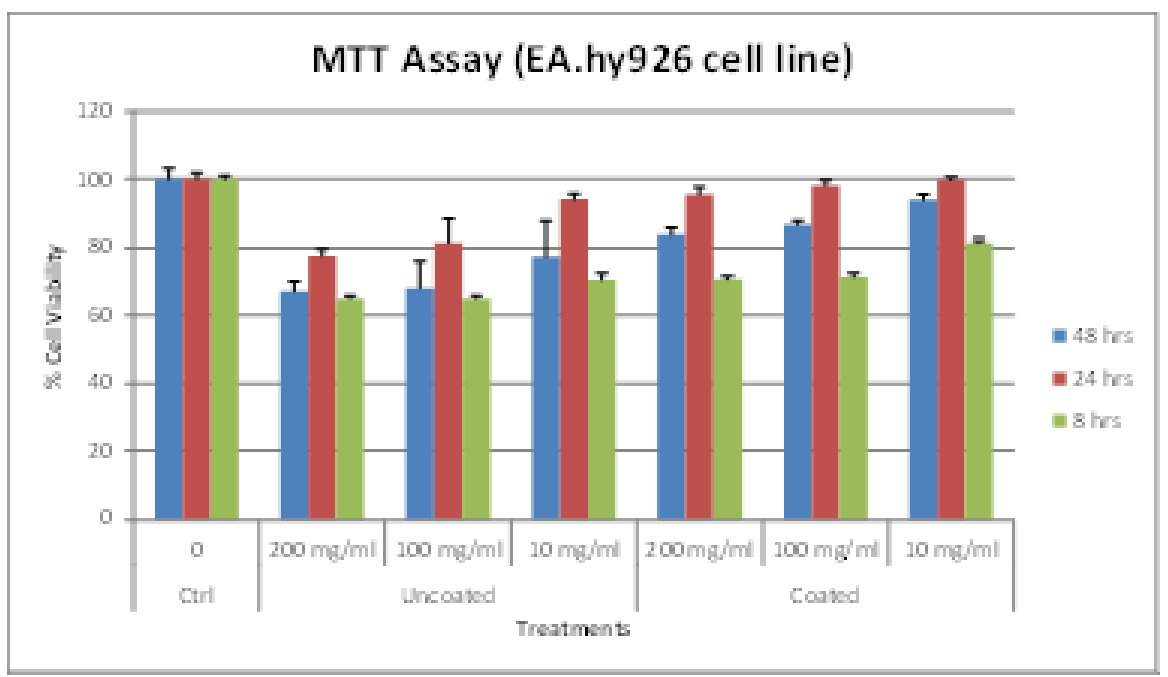

Figure 29: MTT Assay Result.

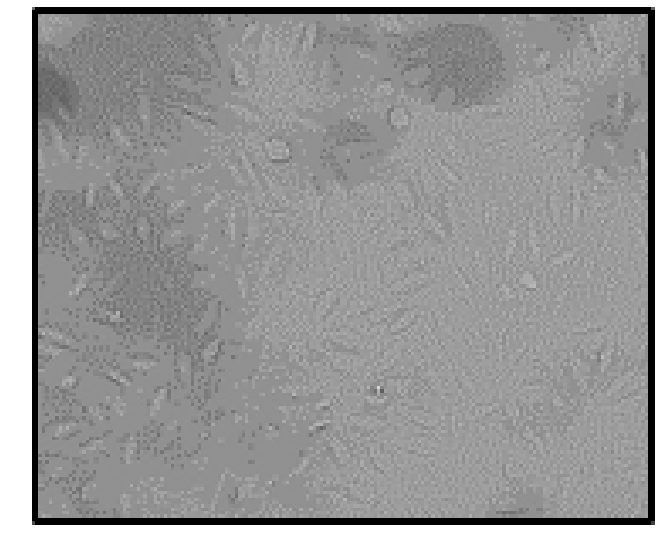

Figure 30: Control 


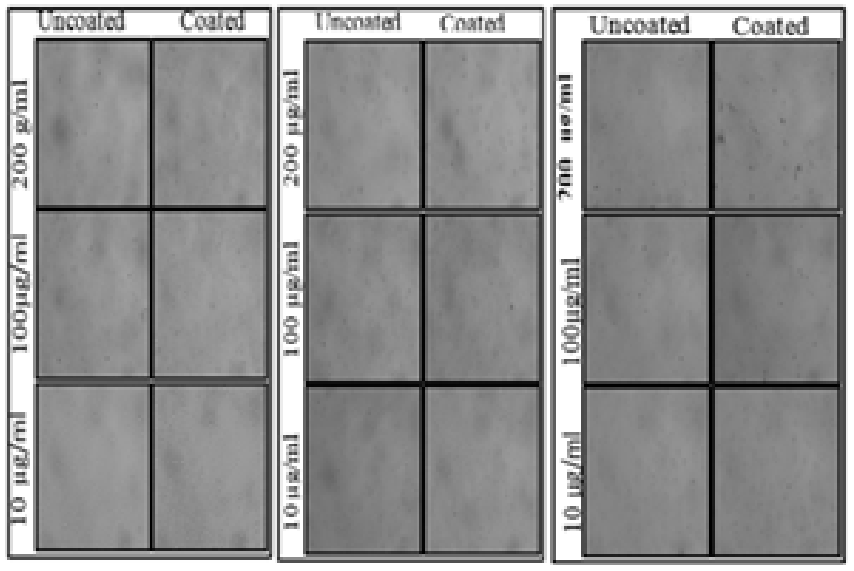

Figure 31: 31-Results of MTT Assay using Microscopes in the duration of (a) 8hrs, (b) 24hrs, (c) 48hrs.

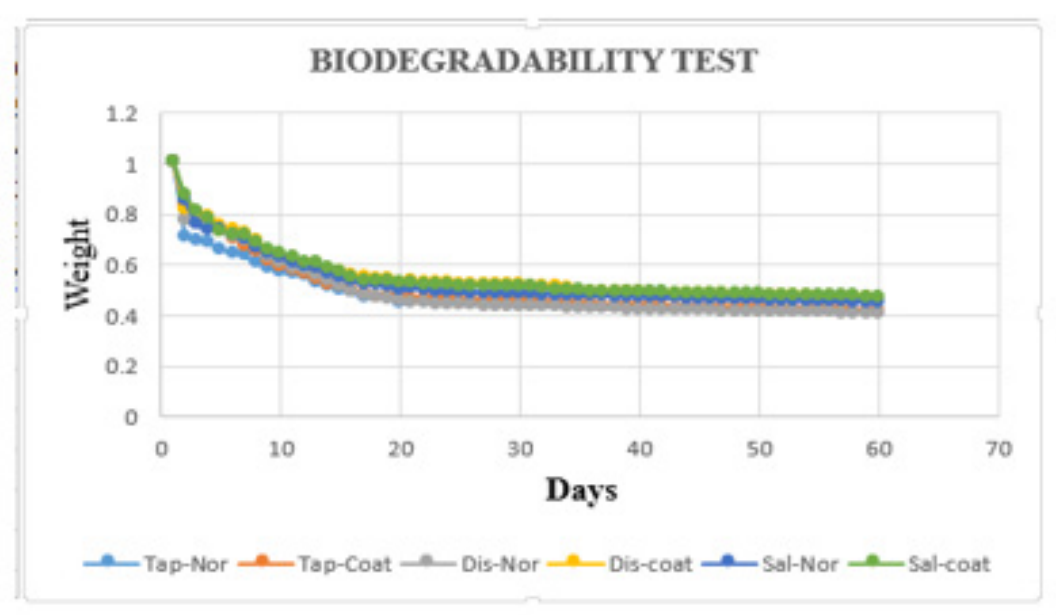

Figure 32: Daily Graph.

\section{Biodegradability}

In this work, the biodegradability was checked through the fluids, because at the end the product is going to be applied to the skin, which consists of the majority of fluids. To check that three different fluids (tap water, distilled water, and saline water) were taken. Based on the PH values these fluids were chosen. Two sets of samples were checked. In one set the weight was measured in the daily bases. In another set weights were measured in the interval of two days. In both, the sets sterile and coated tilapia skins were under observation for 60 days. The data set is shown in below table.

Table 1: FTIR interpretation table.
It is clearly shown that in the tap water the sample degraded slowly when compared to distilled water and saline water. The saline water took more time to degrade. One more consideration is that the coated one took more time to degrade when compared with the normal one.

\section{Biodegradability test in a daily basis}

Itis done in two different sets. One in a daily manner. Another in two days' time interval. The dataset which were taken daily shown in below (Table 2)

\begin{tabular}{|c|c|c|}
\hline Range of Spectrum & Stretch & Group \\
\hline $3500-3300 \mathrm{~cm}^{-1}$ & N-H stretch & amines \\
\hline $3500-3200 \mathrm{~cm}^{-1}$ & O-H stretch & Alcohols, a broad, strong band \\
\hline $3100-3000 \mathrm{~cm}^{-1}$ & C-H stretch & Alkenes \\
\hline $3000-2850 \mathrm{~cm}^{-1}$ & C-H stretch & Alkanes \\
\hline $1760-1665 \mathrm{~cm}^{-1}$ & C=O stretch & Ketones, aldehydes, esters \\
\hline $1680-1640 \mathrm{~cm}^{-1}$ & C=C stretch & Alkenes \\
\hline
\end{tabular}


Table 2:

\begin{tabular}{|c|c|c|c|c|c|c|}
\hline Da ys & Tap-Nor & Tap -Coat & Dis-Nor & Dis -Coat & Sal-Nor & Sal -Coat \\
\hline 1 & 1.0047 & 1.0071 & 1.0045 & 1.0049 & 1.009 & 1.0085 \\
\hline 2 & 0.7135 & 0.8417 & 0.7717 & 0.8151 & 0.8536 & 0.874 \\
\hline 3 & 0.6923 & 0.8045 & 0.7573 & 0.8052 & 0.7594 & 0.8132 \\
\hline 4 & 0.6875 & 0.7833 & 0.7416 & 0.7903 & 0.7369 & 0.7802 \\
\hline 5 & 0.6573 & 0.7377 & 0.7297 & 0.7551 & 0.7373 & 0.7351 \\
\hline 6 & 0.6456 & 0.7063 & 0.7134 & 0.7376 & 0.7198 & 0.7211 \\
\hline 7 & 0.6366 & 0.6767 & 0.6978 & 0.7241 & 0.7065 & 0.7174 \\
\hline 8 & 0.6087 & 0.6535 & 0.6741 & 0.6987 & 0.6691 & 0.6912 \\
\hline 9 & 0.5895 & 0.6196 & 0.634 & 0.6533 & 0.6459 & 0.659 \\
\hline 10 & 0.5703 & 0.597 & 0.6097 & 0.6366 & 0.6305 & 0.6475 \\
\hline 11 & 0.564 & 0.5792 & 0.5891 & 0.6145 & 0.6068 & 0.6337 \\
\hline 12 & 0.5561 & 0.5651 & 0.5787 & 0.5991 & 0.5915 & 0.61 \\
\hline 13 & 0.5324 & 0.5467 & 0.5598 & 0.5872 & 0.5855 & 0.6087 \\
\hline 14 & 0.5156 & 0.5254 & 0.5369 & 0.5733 & 0.5684 & 0.591 \\
\hline 15 & 0.5003 & 0.5152 & 0.5151 & 0.5702 & 0.5513 & 0.5702 \\
\hline 16 & 0.4928 & 0.5044 & 0.4968 & 0.5618 & 0.5352 & 0.5516 \\
\hline 17 & 0.472 & 0.4864 & 0.4775 & 0.5519 & 0.5232 & 0.5399 \\
\hline 18 & 0.4698 & 0.471 & 0.4728 & 0.5423 & 0.5207 & 0.538 \\
\hline 19 & 0.4686 & 0.4692 & 0.4646 & 0.5415 & 0.5146 & 0.5347 \\
\hline 20 & 0.4505 & 0.4685 & 0.4597 & 0.532 & 0.5001 & 0.5288 \\
\hline 21 & 0.4577 & 0.467 & 0.4514 & 0.5352 & 0.5051 & 0.5264 \\
\hline 22 & 0.4563 & 0.4637 & 0.4498 & 0.5332 & 0.5028 & 0.5254 \\
\hline 23 & 0.4539 & 0.4634 & 0.4448 & 0.5325 & 0.4948 & 0.5238 \\
\hline 24 & 0.4513 & 0.4624 & 0.4433 & 0.5314 & 0.4932 & 0.5222 \\
\hline 25 & 0.4503 & 0.4611 & 0.4424 & 0.526 & 0.4911 & 0.5184 \\
\hline 26 & 0.4482 & 0.4602 & 0.4412 & 0.5249 & 0.4896 & 0.5172 \\
\hline 27 & 0.4462 & 0.4581 & 0.439 & 0.5239 & 0.4881 & 0.5168 \\
\hline 28 & 0.4458 & 0.4576 & 0.4388 & 0.5226 & 0.4879 & 0.5155 \\
\hline 29 & 0.4444 & 0.4565 & 0.4374 & 0.5208 & 0.4865 & 0.5138 \\
\hline 30 & 0.4436 & 0.455 & 0.4358 & 0.5191 & 0.4849 & 0.5126 \\
\hline 31 & 0.4423 & 0.4546 & 0.4347 & 0.5176 & 0.483 & 0.5118 \\
\hline 32 & 0.4416 & 0.4517 & 0.4332 & 0.5151 & 0.4826 & 0.5109 \\
\hline 33 & 0.4487 & 0.4489 & 0.4326 & 0.5138 & 0.4811 & 0.4997 \\
\hline 34 & 0.4471 & 0.4452 & 0.4318 & 0.5112 & 0.4798 & 0.4991 \\
\hline 35 & 0.4362 & 0.4421 & 0.4295 & 0.497 & 0.4784 & 0.4985 \\
\hline 36 & 0.4347 & 0.4408 & 0.4279 & 0.4948 & 0.4774 & 0.4972 \\
\hline 37 & 0.4333 & 0.4385 & 0.4261 & 0.4933 & 0.4758 & 0.4956 \\
\hline 38 & 0.4324 & 0.4375 & 0.4254 & 0.4926 & 0.4749 & 0.4943 \\
\hline 39 & 0.4317 & 0.4361 & 0.4248 & 0.4911 & 0.4735 & 0.4936 \\
\hline 40 & 0.4302 & 0.4354 & 0.4237 & 0.4903 & 0.4722 & 0.4928 \\
\hline 41 & 0.4392 & 0.4344 & 0.4229 & 0.4891 & 0.4715 & 0.4914 \\
\hline 42 & 0.4289 & 0.4334 & 0.4211 & 0.4889 & 0.4699 & 0.4909 \\
\hline 43 & 0.428 & 0.4322 & 0.4215 & 0.4876 & 0.4686 & 0.4898 \\
\hline 44 & 0.4275 & 0.431 & 0.4207 & 0.4868 & 0.4678 & 0.4889 \\
\hline
\end{tabular}




\begin{tabular}{|l|c|c|c|c|c|c|}
\hline 45 & 0.4267 & 0.4299 & 0.42 & 0.4855 & 0.4666 & 0.4881 \\
\hline 46 & 0.4255 & 0.429 & 0.4188 & 0.4846 & 0.4657 & 0.4873 \\
\hline 47 & 0.4242 & 0.4283 & 0.4179 & 0.4832 & 0.4643 & 0.4861 \\
\hline 48 & 0.422 & 0.4275 & 0.417 & 0.4817 & 0.4633 & 0.4855 \\
\hline 49 & 0.4232 & 0.4262 & 0.4161 & 0.4809 & 0.4629 & 0.4841 \\
\hline 50 & 0.4226 & 0.4257 & 0.4153 & 0.479 & 0.4617 & 0.4833 \\
\hline 51 & 0.4217 & 0.4249 & 0.4146 & 0.4785 & 0.4604 & 0.4825 \\
\hline 52 & 0.4211 & 0.424 & 0.414 & 0.4776 & 0.4592 & 0.4814 \\
\hline 53 & 0.4203 & 0.4232 & 0.4134 & 0.4769 & 0.4585 & 0.4806 \\
\hline 54 & 0.4198 & 0.4221 & 0.4127 & 0.4761 & 0.4574 & 0.4795 \\
\hline 55 & 0.4191 & 0.4217 & 0.4114 & 0.4755 & 0.4566 & 0.4782 \\
\hline 56 & 0.4185 & 0.4209 & 0.4109 & 0.474 & 0.4552 & 0.4777 \\
\hline 57 & 0.4178 & 0.4197 & 0.4101 & 0.4733 & 0.4547 & 0.4768 \\
\hline 58 & 0.4166 & 0.4175 & 0.4084 & 0.4719 & 0.4528 & 0.4757 \\
\hline 59 & 0.4145 & 0.4161 & 0.4072 & 0.4696 & 0.4514 & 0.4744 \\
\hline 60 & 0.4128 & 0.4144 & 0.4065 & 0.4677 & 0.45 & 0.4728 \\
\hline
\end{tabular}

Table 3: Data set with 3 days interval.

\begin{tabular}{|c|c|c|c|c|c|c|}
\hline Days & Tap-Nor & Tap -Coat & Dis-Nor & Dis -Coat & Sal-Nor & Sal -Coat \\
\hline 1 & 1.009 & 1.009 & 1.005 & 1.005 & 1.009 & 1.008 \\
\hline 4 & 0.688 & 0.789 & 0.745 & 0.793 & 0.729 & 0.777 \\
\hline 7 & 0.638 & 0.679 & 0.694 & 0.723 & 0.701 & 0.707 \\
\hline 10 & 0.565 & 0.596 & 0.608 & 0.638 & 0.635 & 0.649 \\
\hline 14 & 0.501 & 0.539 & 0.557 & 0.594 & 0.547 & 0.592 \\
\hline 17 & 0.485 & 0.472 & 0.481 & 0.533 & 0.518 & 0.554 \\
\hline 21 & 0.463 & 0.454 & 0.454 & 0.525 & 0.502 & 0.539 \\
\hline 24 & 0.466 & 0.454 & 0.445 & 0.527 & 0.499 & 0.535 \\
\hline 28 & 0.454 & 0.443 & 0.431 & 0.513 & 0.481 & 0.522 \\
\hline 31 & 0.451 & 0.442 & 0.432 & 0.503 & 0.481 & 0.517 \\
\hline 35 & 0.443 & 0.438 & 0.423 & 0.495 & 0.476 & 0.493 \\
\hline 39 & 0.439 & 0.436 & 0.429 & 0.491 & 0.475 & 0.498 \\
\hline 43 & 0.436 & 0.423 & 0.427 & 0.488 & 0.469 & 0.482 \\
\hline 46 & 0.423 & 0.423 & 0.415 & 0.486 & 0.468 & 0.487 \\
\hline 50 & 0.423 & 0.429 & 0.419 & 0.477 & 0.468 & 0.485 \\
\hline 53 & 0.425 & 0.426 & 0.415 & 0.476 & 0.453 & 0.488 \\
\hline 57 & 0.419 & 0.417 & 0.417 & 0.477 & 0.455 & 0.473 \\
\hline 60 & 0.416 & 0.412 & 0.408 & 0.467 & 0.452 & 0.478 \\
\hline
\end{tabular}

The graphical representation of the six samples in the three different fluids is shown below. It is clearer that the sample in the saline water takes more time to degrade when compared to tap water (Figure 32).

The biodegradability of the sample in the tap water is shown in below graph. In that the orange dots represent the coated sample, and the blue dots denotes the sterile sample. From that graph, it is clear that the coated one take some time to degrade when compared with uncoated one (Figure 33).
The biodegradability of the sample in the distilled water is shown in below graph. In that, the orange dots represent the coated sample, and the blue dots denotes the sterile sample. From that graph, it is clear that the coated one take some time to degrade when compared with uncoated one. The samples in the distilled water degraded slowly when compared with tap water (Figure 34).

The biodegradability of the sample in the saline water is shown in below graph. In that, the orange dots represent the coated sample, and the blue dots denotes the sterile sample. From that 
graph, it is clear that the coated one take some time to degrade when compared with uncoated one. The samples in the distilled water degraded slowly when compared with distilled water and tap water (Figure 35).

\section{Biodegradability test with two days' time interval}

In that, the weights are measured in two days once. And it is compared with the dataset which was taken daily. The two data sets are matched. The dataset which was taken in two-day time interval shown in below (Table 3)

The graphical representation of biodegradability of sample in three different fluids is shown in below. From the graph, it is clear that the samples in the saline water some more time to degrade when compared to tap water and distilled water (Figure 36).

The biodegradability of the sample in the tap water is shown in below graph. In that, the orange dots represent the coated sample, and the blue dots denotes the sterile sample. From that graph, it is clear that the coated one take some time to degrade when compared with uncoated one. It is matched with the dataset which was taken daily (Figure 37).

The biodegradability of the sample in the distilled water is shown in below graph. In that, the orange dots represent the coated sample, and the blue dots denotes the sterile sample. From that graph, it is clear that the coated one take some time to degrade when compared with uncoated one. The samples in the distilled water degraded slowly when compared with tap water. It is matched with the dataset which was taken daily (Figure 38).

The biodegradability of the sample in the saline water is shown in below graph. In that, the orange dots represent the coated sample, and the blue dots denotes the sterile sample. From that graph, it is clear that the coated one take some time to degrade when compared with uncoated one. The samples in the saline water degraded slowly when compared with tap water and distilled water. It is matched with the dataset which was taken daily (Figure 39).

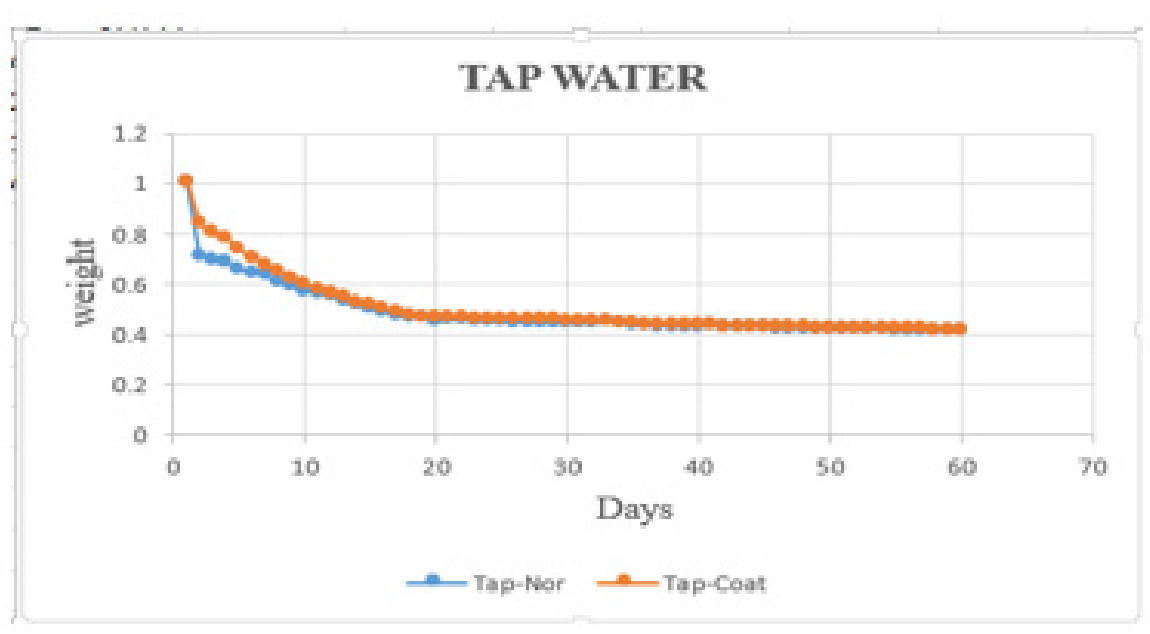

Figure 33: Biodegradability of the Sample in Tap Water.

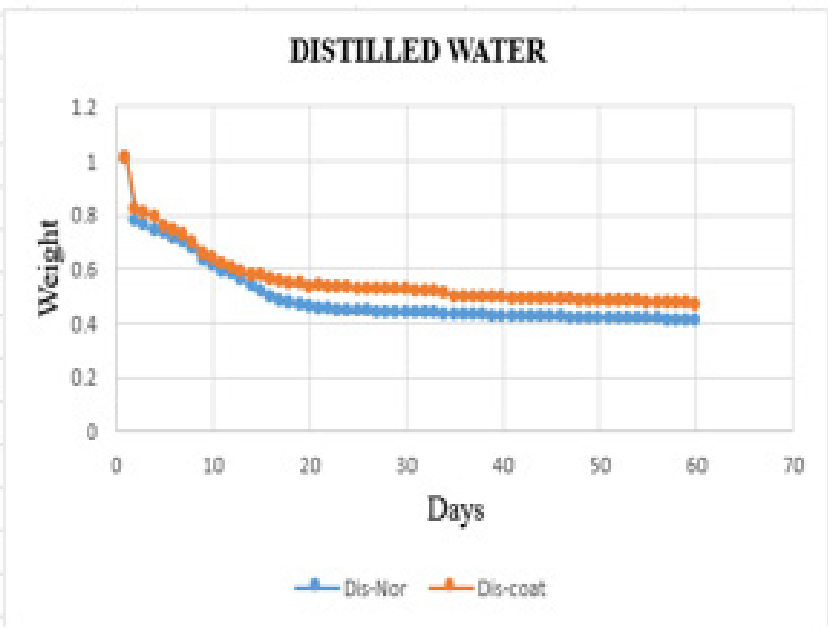

Figure 34: Biodegradability of the Sample in Tap Water. 


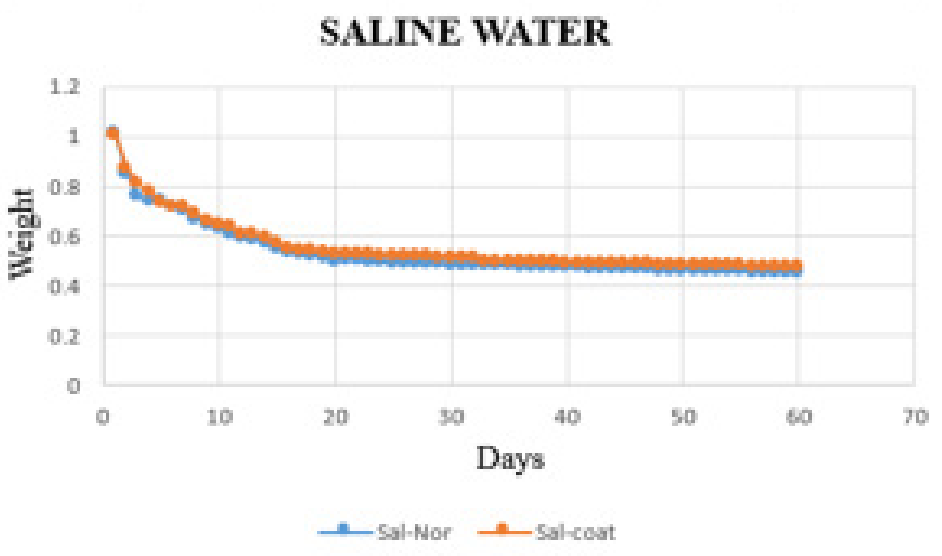

Figure 35: Saline Water.

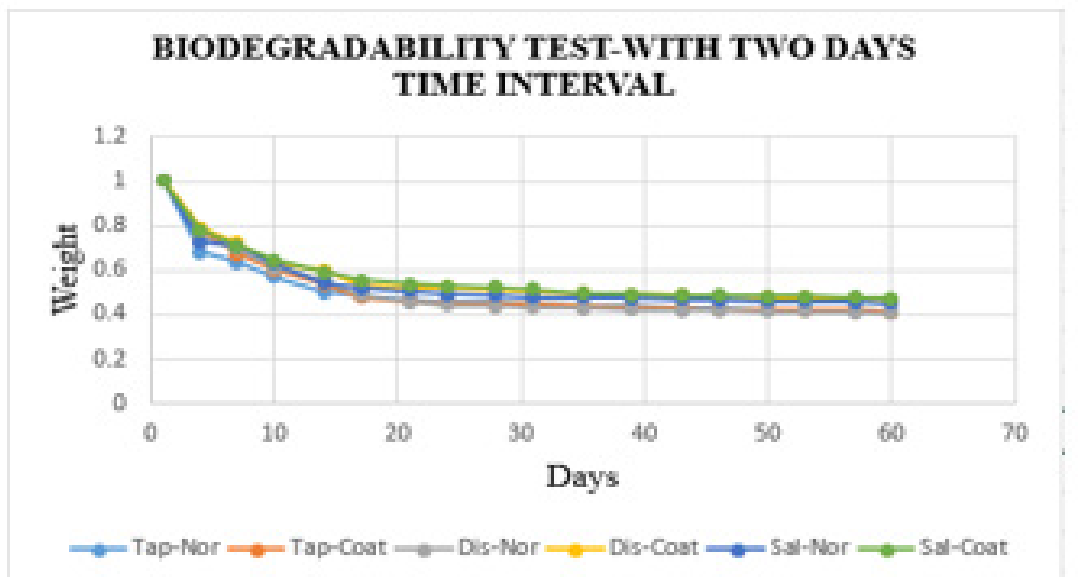

Figure 36: Biodegradability Test with Time Interval.

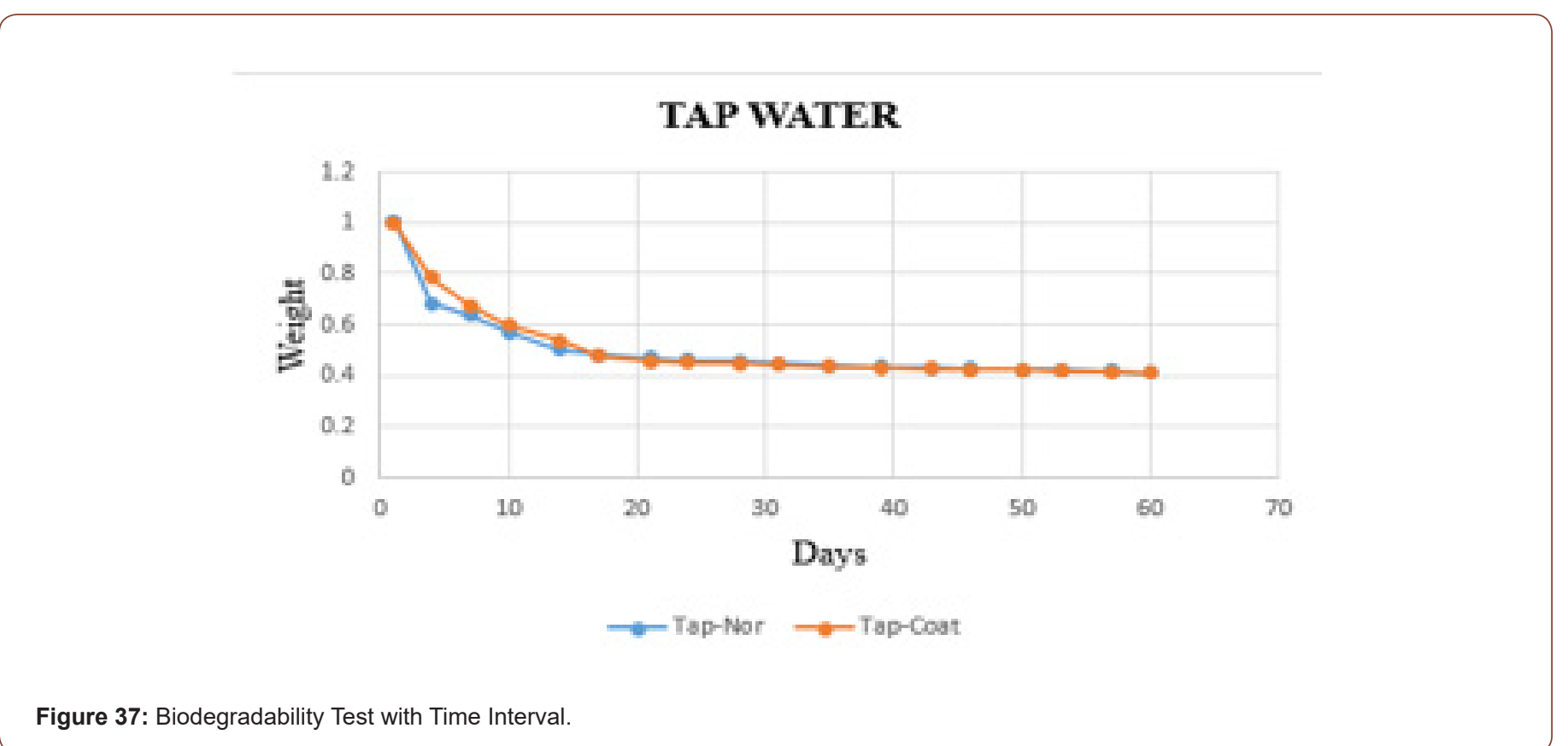




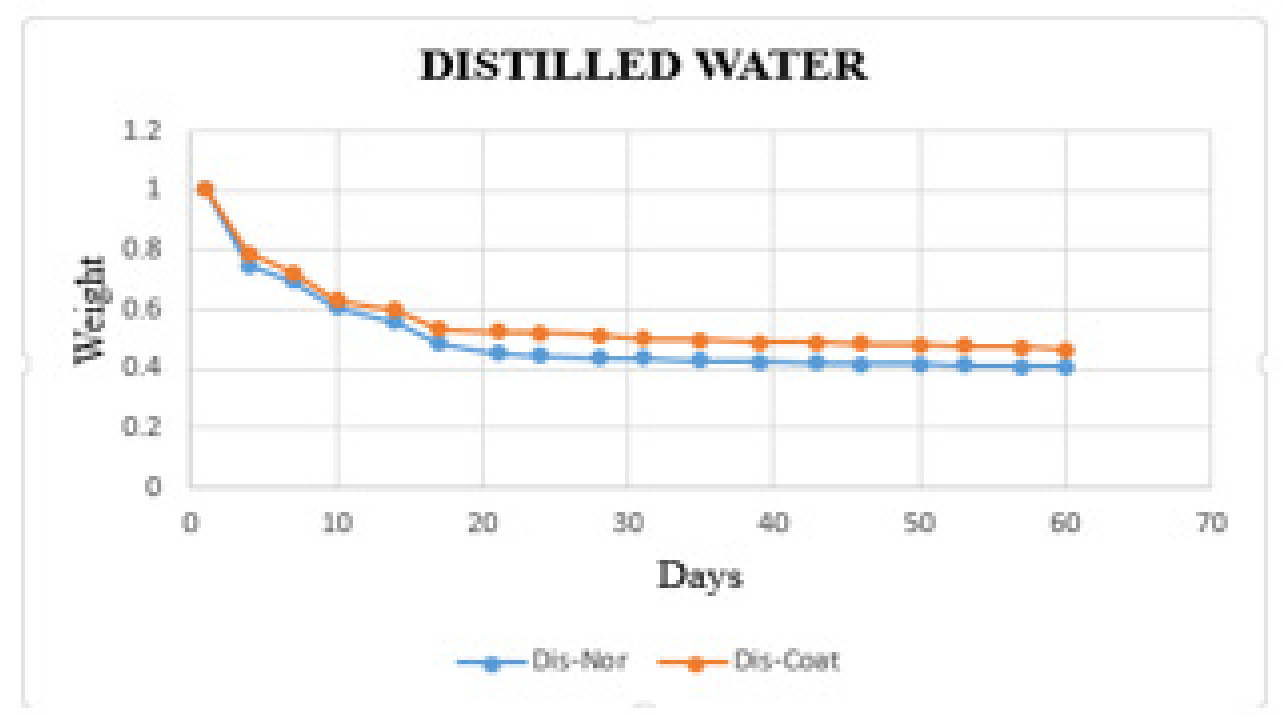

Figure 38: Distilled Water.

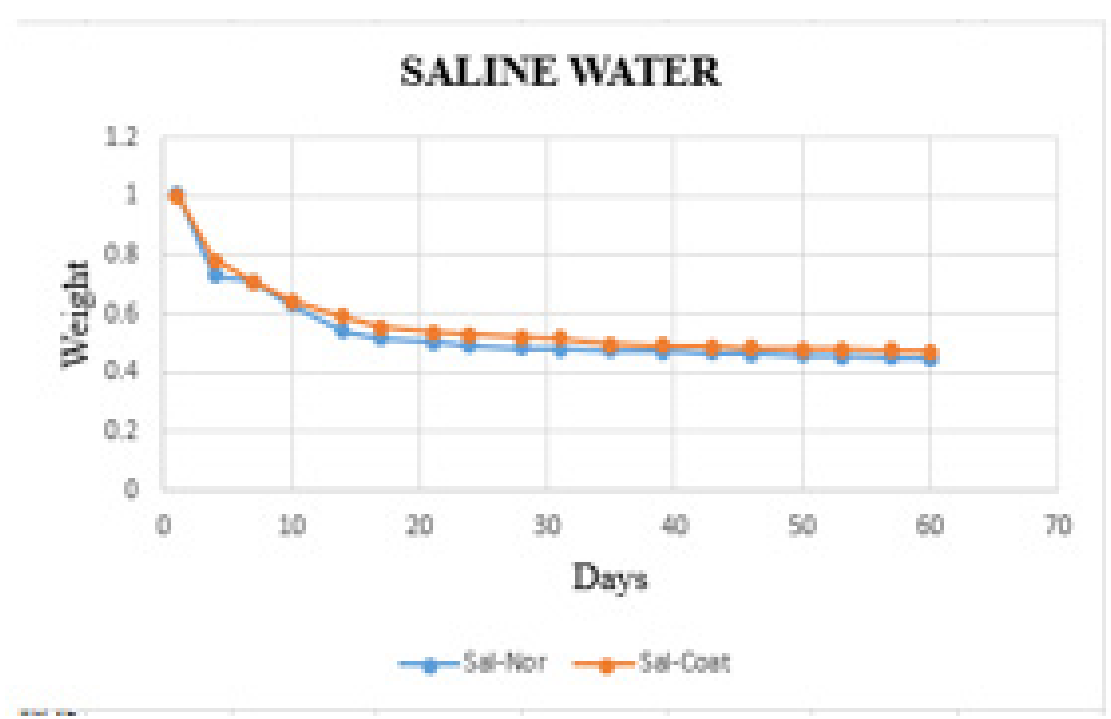

Figure 39: Saline Water.

\section{Conclusion}

Scaffolds from the tilapia skin was prepared by blending the tridax procumbens extract. Stability of the chemical composition studied and confirmed by FTIR studies. Scaffolds tensile strength is calculated as $0.717 \mathrm{MPa}$. It is higher than the tensile strength of medical tape.Its biodegradability is checked in different fluids based on the PH values. Though it is going to be used in contact with the human fluids. The results revealed that it is not break until 60 days. Scaffolds structural morphology is studied by the SEM results. And it shows the fibre bundles, spongicity and tiny porous present in it.Viability of cells that is the cytotoxicity of the sample is checked by the MTT assay method. The coated scaffold has less toxicity when compared with the uncoated scaffold.

\section{Acknowledgment}

None.

\section{Conflicts of Interest}

No conflicts of interest.

\section{References}

1. Edmar Maciel Lima, Filho Manoel Odorico De Moraes, Marcelo Jose Borges De Miranda, Nelson Sarto Piccolo (2017) Tilapia Skin Processing Method and Use thereof for Covering Skin Injuries. Rexresearch, 
2. Gerald T, Lionelli W, Thomas Lawrence (2003) Wound Dressing. Surgical Clinics of North America 83: 617-638.

3. Ruben F Pereira, Cristina C Barrias, Pedro L Granja, Paulo J Bartolo (2013) Advanced biofabrication strategies for skin Regeneration and repair. Advanced nano biomaterials for tissue engineering and regenerative medicine 8(4): 603-621.

4. Xin Zhao, Xiaoming Sun, Lara Yildirimer, Qi Lang, Zhi Yuan (William) Lin, et al. (2017) Cell infiltrative hydrogel fibrous scaffolds for accelerated wound healing. Elsevier 29: 66-77.

5. Sapna Saini, Anju Dhiman, Sanju Nanda (2016) Traditional Indian Medicinal Plants with Potential Wound Healing Activity. IJPSR 7(5): 1809-1819.

6. Sneha J, Mundada, Ruchi Shivhare (2010) Pharmacology of Tridax procumbens a Weed. International Journal of PharmTech Research 1391-1394.

7. Sharma B, Kumar P (2009) Extraction and Pharmacological Evaluation of Some Extracts of Tridax procumbens and Capparis decidua International Journal of Applied Research in Natural Products 1(4): 5-12.

8. SRP Kethamakka, Meena S Deogade (2014) Jayanti Veda (Tridax procumbens) - Unnoticed Medicinal plant by Ayurveda. 2(1): 6-22.

9. Yogesh P Talekar, biswadeep Das, Tania Paul, Deepali Y Talekar, Kishori G Apte, et al. (2012) Evaluation of Wound Healing Potential of Aqueous and Ethanolic Extracts of Tridax Procumbens Linn. In Wistar Rats. Asian Journal of Pharmaceutical and Clinical Research 5(4): 141-145.
10. Tiago H Silva, Joana Moreira-Silva, Ana LP Marques, Alberta Domingues, Yves Bayon, et al. (2014) Marine Origin Collagens and Its Potential Applications. Mar Drugs 12(12): 5881-5901.

11. Falguni Pati, Pallab Datta, Basudam Adhikari, Santanu Dhara, Kuntal Ghosh, et al. (2011) Collagen scaffolds derived from freshwater fish origin and their biocompatibility. Society for Biomaterials 100(4): 10681079 .

12. Zhang Hu, Ping Yang, Chunxia Zhou, Sidong Li, Pengzhi Hong (2017) Marine Collagen Peptides from the Skin of Nile Tilapia (Oreochromis niloticus): Characterization and Wound Healing Evaluation. Marine Drugs 15(4):102

13. Eun Songa, So Yeon Kimb, Taehoon Chunc, Hyun-Jung Byunc, Young Moo Lee (2006) Collagen scaffolds derived from a marine source and their biocompatibility. Elsevier 27(15): 2951-2961.

14. Tian Zhou, Nanping Wang, Yang Xue, Tingting Ding, Xin Liu, et al. (2015) Development of Biomimetic Tilapia Collagen Nanofibers for Skin Regeneration through Inducing Keratinocytes Differentiation and Collagen Synthesis of Dermal Fibroblasts. ACS Applied Materials \& Interfaces 7(5): 3253-3262. 